PHYSICAL REVIEW D 82, 104039 (2010)

\title{
High-order perturbations of a spherical collapsing star
}

\author{
David Brizuela \\ Theoretisch-Physikalisches Institut, Friedrich-Schiller-Universität, Max-Wien-Platz 1, 07743 Jena, Germany \\ and Instituto de Estructura de la Materia, CSIC, Serrano 121-123, 28006 Madrid, Spain \\ José M. Martín-García \\ Institut d'Astrophysique de Paris, Université Pierre et Marie Curie, CNRS, 98bis Boulevard Arago, 75014 Paris, France, \\ Laboratoire Univers et Théories, CNRS, Université Paris Diderot, 5 Place Jules Janssen, 92190 Meudon, France \\ and Instituto de Estructura de la Materia, CSIC, Serrano 121-123, 28006 Madrid, Spain \\ Ulrich Sperhake \\ Institut de Ciències de l'Espai (CSIC-IEEC), Campus UAB, E-08193 Bellaterra, Spain, \\ Theoretical Astrophysics 350-17, California Institute of Technology, Pasadena, California 91125, \\ and Department of Physics and Astronomy, The University of Mississippi, University, Mississippi 38677, USA
}

\section{Kostas D. Kokkotas}

Theoretical Astrophysics, Eberhard-Karls University of Tübingen, 72076 Tübingen, Germany (Received 28 September 2010; published 17 November 2010)

\begin{abstract}
A formalism to deal with high-order perturbations of a general spherical background was developed in earlier work [D. Brizuela, J. M. Martín-García, and G. A. Mena Marugán, Phys. Rev. D 74, 044039 (2006); D. Brizuela, J. M. Martín-García, and G. A. Mena Marugán, Phys. Rev. D 76, 024004 (2007)]. In this paper, we apply it to the particular case of a perfect fluid background. We have expressed the perturbations of the energy-momentum tensor at any order in terms of the perturbed fluid's pressure, density, and velocity. In general, these expressions are not linear and have sources depending on lower-order perturbations. For the second-order case we make the explicit decomposition of these sources in tensor spherical harmonics. Then, a general procedure is given to evolve the perturbative equations of motions of the perfect fluid for any value of the harmonic label. Finally, with the problem of a spherical collapsing star in mind, we discuss the high-order perturbative matching conditions across a timelike surface, in particular, the surface separating the perfect fluid interior from the exterior vacuum.
\end{abstract}

DOI: 10.1103/PhysRevD.82.104039

PACS numbers: 04.25.Nx, 04.30.Db, 04.40.Dg

\section{INTRODUCTION}

The detection of gravitational waves is currently considered to be one of the most important open problems in astrophysics and experimental physics. Aside from providing a test of the theory of general relativity in the strongfield regime, it will open a new window for astrophysical observations, giving rise to the era of gravitational-wave astronomy. To this end the ground-based laserinterferometric detectors LIGO, VIRGO, GEO600, and TAMA [1-4] are now collecting data at or near design sensitivity, while next generation ground-based detectors like the Einstein Telescope (ET) are in the research and development phase [5]. The space-based interferometer LISA [6], scheduled for launch in the next decade, will complement such observations with high precision in the low-frequency range. Gravitational-wave detection and interpretation requires a close interplay between the experimental side and theoretical predictions as physical signals need to be dug out from a noisy data stream. Various astrophysical scenarios are expected to generate gravitational radiation that will be detectable on Earth. Most prominently among these feature the inspiral and coalescence of compact binaries, black holes, and/or neutron stars, as well as stellar collapse. The work of this article is primarily addressed at the modeling of the latter type of sources although we believe our results to be of interest for a variety of questions pertaining to gravitational-wave source modeling.

Simulating neutron stars and black holes in the framework of fully nonlinear general relativity is only possible using numerical methods on supercomputers, an area of research commonly referred to as numerical relativity. This field has made enormous progress recently; see, for example, the reviews [7-9]. In spite of this progress, approximative techniques such as the post-Newtonian expansion and perturbation theory still play a crucial role in the modeling of astrophysical sources of gravitational waves. First, numerical simulations are computationally too expensive to cover more than a few tens of orbits in inspirals, so that complete waveforms are now constructed by hybridization of numerical and post-Newtonian results $[10,11]$ or calibration of the effective-one-body model via numerical relativity simulations [12-14]. Second, other types of sources completely elude a full numerical treatment. For example, simulations of the so-called extreme 
mass ratio binaries are practical only in a perturbative framework; see [15,16] and references therein for a discussion as well as [17-20] for the most extreme mass ratios simulated in numerical relativity. Perturbation theory also provides a natural framework for interpreting numerical results as, for example, in the case of black-hole and neutron-star oscillations [21,22]. Finally perturbative calculations efficiently provide results with high precision even when involving numerical methods and are thus ideally suited for the study of a variety of astrophysical and cosmological problems as well as benchmarking the accuracy of full blown numerical simulations.

The purpose of this work is to provide a general gaugeinvariant framework for perturbation theory of arbitrary order on time-dependent and spherically symmetric perfect fluid backgrounds. This work constitutes a particularization of the formalism developed in Refs. $[23,24]$ by including matter obeying perfect fluid equations of state. In particular, we will also include a careful analysis of the matching conditions necessary to obtain a consistent treatment of the stellar interior and the vacuum exterior spacetime.

Static backgrounds represent a particularly simple subclass of configurations because they allow for a Fourier expansion of the perturbations in such a way that reduces the problem to a set of ordinary differential equations depending on radius only. Examples for the general type of background configurations considered in this work include radially collapsing or exploding stars. The collapse of homogeneous dust spheres studied extensively in a series of papers by Cunningham, Price, and Moncrief [25-27] can be viewed as the special case of a perfect fluid with vanishing pressure. In this case the different layers of the star do not interact, which leads to a simpler type of motion but may result in unphysical consequences.

The perturbations can be either radial or nonradial, although in some scenarios it turns out to be convenient to absorb radial perturbations in the spherically symmetric background configuration. See, for example, Ref. [28] for a fully nonlinear numerical treatment of radial pulsations of neutron stars. Nonlinearities are naturally incorporated in perturbation theory by including higher-order terms in the calculation. First-order perturbation theory, for example, linearizes the Einstein equations in the first-order metric perturbations ${ }^{\{1\}} h_{\mu \nu}$, whereas second-order theory results in a set of differential equations linear in the second-order metric perturbations ${ }^{\{2\}} h_{\mu \nu}$ but containing source terms quadratic in the ${ }^{\{1\}} h_{\mu \nu}$. This hierarchical structure can be extended to arbitrary order although the equations quickly become overwhelmingly complicated.

The application of perturbation theory to compact stars in a general relativistic framework was pioneered in the 1960s by Chandrasekhar's analysis of the stability of static background models against radial perturbations [29,30]. Next, Thorne and collaborators established the theoretical basis for the study of nonradial perturbations of perfect fluid stars [31-35]. Further development and applications can be found in Refs. [36-39]. Time-dependent background configurations were first studied in the above mentioned work by Cunningham et al. $[25,26]$ on collapsing dust spheres. Seidel and coworkers [40-42] applied the formalism of Gerlach and Sengupta [43,44] to generalize the work of Cunningham et al. to general perfect fluid equations of state. Motivated by this work, Gundlach and Martín-García [45] developed a covariant and gaugeinvariant framework to analyze arbitrary first-order perturbations of a spherical perfect fluid. This framework was later used by Harada et al. [46] to analyze axial perturbations of stellar collapse.

To our knowledge higher-order perturbation theory of stellar models has so far only been applied to the modeling of slowly, uniformly rotating stars. The key idea here is to incorporate the rotation in the form of a first-order axial perturbation. This idea has again been pioneered in the Cunningham et al. series [27] in the special case of a collapsing dust sphere. Applications to a wider class of perfect fluids have so far been restricted to static background models $[47,48]$. Differential rotation has been modeled in the context of the Cowling approximation ${ }^{1}[49,50]$. More recently it has become possible to study perturbations of fast rotating axisymmetric configurations, still in the Cowling approximation [51-53].

Our work represents the natural extension of the existing literature by constructing a general framework for secondorder perturbations on a time-dependent, sphericallysymmetric background spacetime containing matter in the form of a perfect fluid star. The starting point for this construction is given by the second-order generalization of the Gerlach-Sengupta formalism developed in Refs. [23,24]. Specifically, we will apply this gaugeinvariant formalism to the case of a perfect fluid star following the notation and techniques presented in Ref. [45].

A key ingredient in our study is the matching at second perturbative order of the stellar interior and the vacuum exterior parts of the spacetime. Its first-order analogue was analyzed in a covariant and gauge-invariant manner in [54], but we will see that the extension to higher orders represents a nontrivial problem. Its solution requires a careful analysis as to which quantities must be continuous across the stellar surface. Our analysis of this particular point will be valid at any perturbative order and we believe it will also be helpful in clarifying the matching conditions in certain background configurations.

A brief overview of the quantities and the equations governing their behavior is given as follows. The background metric in the stellar interior is described by the

\footnotetext{
${ }^{1}$ In this approximation one neglects metric perturbations and thus evolves fluid perturbations only.
} 
scalars $U$ and $W$ defined in Eq. (23) and the structure coefficients $\mu$ and $\nu$ given in Eq. (21), all defined with respect to the background velocity field. The fluid is described by its density $\rho$, the entropy per fluid element $s$, and the pressure $p$. These variables are determined by Eqs. (24)-(31) and the equation of state that provides $p$ as a function of $\rho$ and $s$.

The perturbations at order $n$ are naturally divided into axial and polar modes and further decomposed into multipoles with labels $l$ and $m$. For simplicity, we will omit these labels from the perturbation variables in this summary as is also done below in the derivation. The axial perturbations are given by the velocity component $\beta$ (41) and the Gerlach-Sengupta master scalar П (68) which are evolved according to Eqs. (73) and (64). The metric components $\delta$ and $\lambda(61)$ can be reconstructed from the master function $\Pi$ according to Eqs. (70) and (71). The polar perturbations are given by the velocity components $\alpha$ and $\gamma(41)$, the energy and entropy perturbations $\omega$ and $\sigma$ (34) and (35), the metric perturbations $\eta, \chi$, and $\psi$ defined in Eqs. (62) and (63), and the scalar $\mathcal{K}$ (12). This set of perturbations obeys the evolution system (91)-(97) and (106).

The remainder of this paper is organized as follows. Section II presents a brief summary of the generalized formalism introduced in Ref. [23,24]. In Sec. III we introduce the notation for the background fluid variables and their perturbations. We further express the perturbation of the energy-momentum tensor in terms of these variables. The evolution equations resulting from the perturbed Einstein and matter equations are presented in Sec. IV. The high-order matching conditions are the subject of Sec. V. This section is quite independent from the previous ones, so the interested reader is referred directly there. We conclude in Sec. VI with a discussion of future applications and extensions of our work.

The intensive tensor computations in this work have been performed with the tensor computer algebra framework xAct [55], based on Mathematica. Of particular importance has been the package xPert [56] for high-order metric perturbation theory around curved backgrounds.

\section{HIGH-ORDER GERLACH AND SENGUPTA FORMALISM}

\section{A. Background spherical spacetime}

The spherically symmetric nature of the background spacetime enables us to naturally decompose it as $\mathcal{M} \equiv$ $\mathcal{M}^{2} \times S^{2}$, where $\mathcal{M}^{2}$ is a two-dimensional Lorentzian manifold and $S^{2}$ the unit two-sphere. In order to distinguish tensor fields residing on these different manifolds, we will use Greek letters $(\mu, \nu, \ldots)$ for four-dimensional indices, capital Latin letters $(A, B, \ldots)$ for indices on $\mathcal{M}^{2}$, and lowercase Latin letters $(a, b, \ldots)$ for indices on the sphere. With this notation, we can decompose the background metric and energy-momentum tensor as

$$
\begin{gathered}
g_{\mu \nu}\left(x^{D}, x^{d}\right) d x^{\mu} d x^{\nu}= \\
g_{A B}\left(x^{D}\right) d x^{A} d x^{B} \\
+r^{2}\left(x^{D}\right) \gamma_{a b}\left(x^{d}\right) d x^{a} d x^{b}, \\
t_{\mu \nu}\left(x^{D}, x^{d}\right) d x^{\mu} d x^{\nu}= \\
t_{A B}\left(x^{D}\right) d x^{A} d x^{B} \\
+\frac{1}{2} r^{2}\left(x^{D}\right) Q\left(x^{D}\right) \gamma_{a b}\left(x^{d}\right) d x^{a} d x^{b},
\end{gathered}
$$

where $g_{A B}$ is the metric on $\mathcal{M}^{2}, \gamma_{a b}$ is the standard metric on the unit sphere, and $r, Q$ are scalar fields on $\mathcal{M}^{2}$. We denote covariant derivatives associated with the different metrics by

$$
g_{\mu \nu ; \lambda}=0, \quad g_{A B \mid C}=0, \quad \gamma_{a b: c}=0,
$$

and define the vector $v_{A} \equiv r_{\mid A} / r$. The Einstein equations for the metric (1) with matter tensor (2) are given in [43].

Tensor fields of any rank $s$ on the sphere will be decomposed using a basis of tensor spherical harmonics labeled by multipole indices $l$ and $m$. Such basis can be constructed from the symmetric trace-free (STF) tensors

$$
\begin{aligned}
Z_{l \quad a_{1} \ldots a_{s}}^{m} & \equiv\left(Y_{l}^{m}: a_{1} \ldots a_{s}\right)^{\mathrm{STF}}, \\
X_{l a_{1} \ldots a_{s}}^{m} & \left.\equiv \epsilon_{\left(a_{1}\right.}{ }^{b} Z_{l}^{m} b a_{2} \ldots a_{s}\right)
\end{aligned}
$$

which are, respectively, polar and axial, together with the metric $\gamma_{a b}$ and the antisymmetric tensor $\epsilon_{a b}$ [23]. For the particular case $s=0$, those objects must be read as $Z_{l}^{m} \equiv$ $Y_{l}^{m}$ and $X_{l}^{m} \equiv 0$.

At first order modes with different $l, m$ and polarities decouple. However, at second and higher perturbative orders, the nonlinear coupling between first-order modes results in products of tensor spherical harmonics with different labels $(\hat{l}, \hat{m}, \hat{s})$ and $(\bar{l}, \bar{m}, \bar{s})$. Those products can be decomposed into a linear combination of harmonics $(l$, $\hat{m}+\bar{m}, \hat{s}+\bar{s}$ ) with an explicit formula involving coefficients

$$
E_{\bar{s} \bar{s} \hat{l} \bar{m} l}^{\hat{l} \hat{m}} \propto C_{\bar{l} \hat{l} l}^{\bar{m} \hat{m} \hat{m}+\bar{m}} C_{\bar{l} \hat{s} \hat{l} \hat{s} \hat{s}+\bar{s}}^{\bar{l}}
$$

where $C$ stands for Clebsch-Gordan coefficients. These $E$-coefficients encode the geometric selection rules that determine which pairs of modes do actually couple. See [23] for full details.

\section{B. Nonspherical perturbations}

Perturbative calculations are often formulated in terms of a one-parameter family of spacetimes $(\mathcal{M}(\varepsilon), g(\varepsilon))$. The expansion parameter represents a measure for how strongly a physical system deviates from the background configuration corresponding to $\varepsilon=0$. In complete analogy, any tensorial quantity of the system is represented by a family $\Omega(\varepsilon)$, and expanded around its background value $\Omega \equiv \Omega(\varepsilon=0)$ according to 


$$
\Omega(\varepsilon)=\Omega+\sum_{n=1}^{\infty} \varepsilon^{n} \frac{\Delta^{n}[\Omega]}{n !} .
$$

A key feature of this expansion is that the perturbations $\Delta^{n}[\Omega]$, also denoted by ${ }^{\{n\}} \Omega$ in this work, are tensors on the background manifold. We have thus converted a single problem on an unknown manifold into an infinite hierarchy of problems on the chosen background manifold. There remains, however, one important problem; we need to

specify a diffeomorphism $\phi(\varepsilon)$ relating points on the background $\mathcal{M}$ and the perturbed manifolds $\mathcal{M}(\varepsilon)$. The actual values of the perturbations ${ }^{\{n\}} \Omega$ depend on this choice of gauge.

We next explicitly decompose tensorial perturbations in terms of the basis of tensor harmonics introduced in Eqs. (4) and (5). In this expansion the metric perturbations are given by,

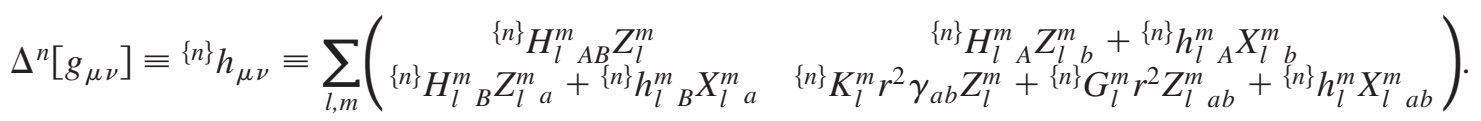

At each perturbative order $n$, the gauge freedom enables us to choose freely four of these functions, three polar and one axial. Alternatively to thus fixing the gauge, we can construct gauge-invariant combinations of the perturbation functions and work with those quantities. We will follow this latter approach, though with a construction based on the choice of a particular gauge.

For this purpose we consider the most natural gauge choice in spherical symmetry: Regge-Wheeler (RW) gauge [57]. It is defined by setting

$$
{ }^{\{n\}} H_{l}^{m}{ }_{A}=0, \quad{ }^{\{n\}} G_{l}^{m}=0, \quad{ }^{\{n\}} h_{l}^{m}=0,
$$

for all $n \geq 1$ and $l \geq 2$. We note that this does not constitute a rigid choice, that is, there remains some gauge freedom in the $l=0$ and $l=1$ modes. Next we use an important result from Ref. [24], namely, a procedure to define gauge-invariant quantities whose values coincide with those of the perturbations in RW gauge. The key idea of this procedure is to employ the formula for general gauge transformations parametrized by the generators $\{n\} \xi$, changing the perturbation $\Delta^{n}[\Omega]$ to

$$
\begin{aligned}
& \Delta^{n}[\Omega]+\sum_{m=1}^{n} \frac{n !}{(n-m) !} \sum_{\left(K_{m}\right)} \frac{1}{2 !^{k_{2}} \ldots(m !)^{k_{m}} k_{1} ! \ldots k_{m} !} \\
& \quad \times \mathcal{L}_{\{1\} \xi}^{k_{1}} \ldots \mathcal{L}_{\{m\} \xi}^{k_{m}} \Delta^{n-m}[\Omega],
\end{aligned}
$$

where the second sum extends to the $m$-tuples

$$
\left(K_{m}\right)=\left\{\left(k_{1}, \ldots, k_{m}\right) \in \mathbb{N}_{0}^{m} ; \sum_{j=1}^{m} j k_{j}=m\right\},
$$

$\mathbb{N}_{0}$ being the set of non-negative integers. We then need to choose the vectors ${ }^{\{m\}} \xi$ such that they take the metric perturbation (8) from an arbitrary gauge to the RW form (9). Naturally, the vectors ${ }^{\{m\}} \xi$ depend on the perturbations ${ }^{\{k\}} h_{\mu \nu}$ themselves, and so the expressions for the gauge invariants are highly nontrivial beyond first order. Explicit formulas at second order, already expanded in terms of the harmonic coefficients, are given in [24].

Given that the perturbations in RW gauge and the associated gauge invariants coincide in value, we will perform all our computations in RW gauge, for simplicity, and then use the expressions in [24] whenever we want to generalize the results to an arbitrary gauge.

In this way, the decomposition of the perturbations of the metric and the energy-momentum tensor will be as follows:

$$
\begin{aligned}
& { }^{\{n\}} h_{\mu \nu} \equiv \sum_{l, m}\left(\begin{array}{cc}
{ }^{\{n\}} \mathcal{K}_{l A B}^{m} Z_{l}^{m} & \{n\} \kappa_{l A}^{m} X_{l b}^{m} \\
{ }^{\{n\}} \kappa_{l B}^{m} X_{l a}^{m} a & \{n\} \mathcal{K}_{l}^{m} r^{2} \gamma_{a b} Z_{l}^{m}
\end{array}\right)
\end{aligned}
$$

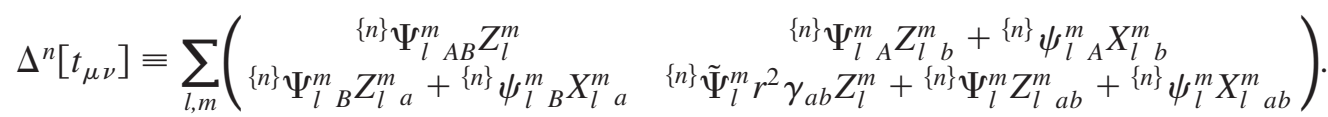

Note that polar (axial) harmonic coefficients are represented by uppercase (lowercase) letters. In order to agree with the construction by Gerlach and Sengupta [43,44], we combine the invariants ${ }^{\{n\}} \psi_{l_{A}}^{m}$ and ${ }^{\{n\}} \kappa_{l A}^{m}$ to form a new matter invariant quantity

$$
\{n\} \tilde{\psi}_{l A}^{m} \equiv\{n\} \psi_{l A}^{m}-\frac{Q^{\{n\}}}{2} \kappa_{l A}^{m}
$$




\section{PERFECT FLUID}

The decomposition of metric and matter perturbations given above is valid for arbitrary types of matter sources. In this section we discuss in detail the special case of a perfect fluid. We first formulate the equations for the background spacetime in Sec. III A, following [45]. Then we address the description of fluid perturbations in Sec. III B, generalizing the notations and results in [45] from first to higher orders. The metric and fluid perturbative evolution equations will be analyzed in the next section.

\section{A. Background perfect fluid}

The energy-momentum tensor for a perfect fluid with four-velocity $u^{\mu}$, total energy density $\rho$, and pressure $p$ is

$$
t_{\mu \nu} \equiv(\rho+p) u_{\mu} u_{\nu}+p g_{\mu \nu} .
$$

This corresponds to the special case $Q=2 p$ in the general expression (2). We will use a generic equation of state for a perfect fluid, in the form $p=p(\rho$, $s)$ where $s$ is the entropy per particle. It is convenient to introduce the partial derivatives of the pressure as additional quantities according to

$$
c_{s}^{2} \equiv\left(\frac{\partial p}{\partial \rho}\right)_{s}, \quad C \equiv \frac{1}{\rho}\left(\frac{\partial p}{\partial s}\right)_{\rho} .
$$

Here, $c_{s}^{2}$ is the adiabatic speed of sound, but we are not aware of a comparatively intuitive meaning of the second quantity.

In spherical symmetry the four-velocity of the fluid takes the form $u^{\mu}=\left(u^{A}, 0\right)$. This vector defines a unique outward pointing spacelike unit vector $n_{A} \equiv-\epsilon_{A B} u^{B}$ on $\mathcal{M}^{2}$, with $\epsilon_{A B}$ being the antisymmetric volume form on $\mathcal{M}^{2}$. These two vectors form an orthonormal basis on this manifold, which can be used to decompose all geometrical objects, for example

$$
g_{A B}=-u_{A} u_{B}+n_{A} n_{B}, \quad \epsilon_{A B}=n_{A} u_{B}-u_{A} n_{B} .
$$

Using this decomposition, the $\mathcal{M}^{2}$ part of the energymomentum tensor (2) takes the form

$$
t_{A B}=\rho u_{A} u_{B}+p n_{A} n_{B} .
$$

For scalar fields $f$ on $\mathcal{M}^{2}$ we further define the frame derivatives

$$
\dot{f} \equiv u^{A} f_{\mid A}, \quad f^{\prime} \equiv n^{A} f_{\mid A} .
$$

A straightforward calculation shows that the frame derivatives obey the commutation relation

$$
(\dot{f})^{\prime}-\left(f^{\prime}\right)^{\cdot}=\mu f^{\prime}-\nu \dot{f},
$$

where

$$
\mu \equiv u_{\mid A}^{A}, \quad \nu \equiv n_{\mid A}^{A} .
$$

These "structure" functions are the components of the covariant derivatives of the frame vectors in their own frame

$$
u_{A \mid B}=n_{A}\left(n_{B} \mu-u_{B} \nu\right), \quad n_{A \mid B}=u_{A}\left(n_{B} \mu-u_{B} \nu\right) .
$$

We are now in the position to formulate the Einstein equations exclusively in terms of the background scalars $\rho$, $p, \mu, \nu$, and

$$
U \equiv u^{A} v_{A}, \quad W \equiv n^{A} v_{A} .
$$

For our spherically symmetric background we thus obtain expressions with remarkable symmetry for the four independent field equations

$$
\begin{gathered}
U^{\prime}=W(\mu-U), \\
\dot{W}=U(\nu-W), \\
W^{\prime}=-4 \pi \rho-W^{2}+U \mu+\frac{M}{r^{3}}, \\
\dot{U}=-4 \pi p-U^{2}+W \nu-\frac{M}{r^{3}},
\end{gathered}
$$

with the Hawking mass [58] in spherical symmetry (Misner-Sharp mass)

$$
M \equiv \frac{r}{2}\left(1-r_{, A} r^{A}\right)=\frac{r}{2}\left[1+r^{2}\left(U^{2}-W^{2}\right)\right] .
$$

We still need to consider the equations of motion for the perfect fluid. Conservation of energy-momentum results in

$$
\begin{gathered}
\dot{\rho}+(\rho+p)(2 U+\mu)=0, \\
c_{s}^{2} \rho^{\prime}+\rho C s^{\prime}+(\rho+p) \nu=0,
\end{gathered}
$$

which represent energy conservation and the Euler equation, respectively. Finally, a perfect fluid does not dissipate energy and hence the entropy of each fluid element is conserved,

$$
\dot{s}=0 .
$$

The system (24)-(31) fully describes the dynamics of our background spacetime. In our derivation of the perturbation equations below, they will further enable us to simplify those coefficients which depend on the background.

\section{B. High-order perfect fluid perturbations}

Our formulation of the fluid perturbations proceeds in two stages. First, we expand the perturbations of the energy-momentum tensor in terms of perturbations ${ }^{\{n\}} \rho$, ${ }^{\{n\}} S$ and ${ }^{\{n\}} \mathcal{U}^{\mu}$ of density, entropy, and four-velocity, respectively. Second, we need to expand the latter as series in appropriate tensor spherical harmonics.

The explicit form of the $n$th perturbation of the energymomentum tensor of the perfect fluid (15) is obtained by using standard combinatorial formulas [56]. The resulting expression is given by 


$$
{ }^{\{n\}} \Psi_{\mu \nu} \equiv \Delta^{n}\left[t_{\mu \nu}\right]=\sum_{k=0}^{n}\left(\begin{array}{l}
n \\
k
\end{array}\right)\left\{\{k\} p^{\{n-k\}} h_{\mu \nu}+\sum_{j=k}^{n}\left(\begin{array}{c}
n-k \\
j-k
\end{array}\right)\left({ }^{\{k\}} \rho+\{k\} p\right)^{\{j-k\}} u_{\mu}{ }^{\{n-j\}} u_{\nu}\right\},
$$

with ${ }^{\{n\}} u_{\mu} \equiv \Delta^{n}\left[u_{\mu}\right]$. This can be further expanded along the same lines, using a high-order generalization of the chain rule on the equation of state,

$$
{ }^{\{n\}} p=\sum \frac{n !}{2 !^{k_{2}} \ldots n !^{k_{n}} 2 !^{r_{2}} \ldots n !^{r_{n}} k_{1} ! \ldots k_{n} ! r_{1} ! \ldots r_{n} !} \frac{\partial^{(K+R)} p}{\partial \rho^{K} \partial s^{R}}{ }^{\{n\}} \rho^{k_{1}} \ldots{ }^{\{n\}} \rho^{k_{n}\{n\}} s^{r_{1}} \ldots s^{r_{n}}
$$

where the sum is restricted to the following $2 n$-tuples:

$$
\left\{\left(k_{1}, \ldots, k_{n}, r_{1}, \ldots, r_{n}\right) \in \mathbb{N}_{0}^{2 n} ; \sum_{j=1}^{n} j\left(k_{j}+r_{j}\right)=n\right\}
$$

and we have defined $K \equiv \sum_{j=1}^{n} k_{j}$ and $R \equiv \sum_{j=1}^{n} r_{j}$. In practice derivatives of the pressure will be replaced by the sound speed $c_{s}$ and thermodynamic factor $C$, defined in Eqs. (16). The expression for the pressure perturbation is completed by the harmonic decomposition of the density and entropy perturbations

$$
\begin{gathered}
{ }^{\{n\}} \rho \equiv \rho \sum_{l, m}{ }^{\{n\}} \omega_{l}^{m} Z_{l}^{m}, \\
{ }^{\{n\}} \equiv \sum_{l, m}{ }^{\{n\}} \sigma_{l}^{m} Z_{l}^{m} .
\end{gathered}
$$

Our formulation of the perturbations of the four-velocity is guided by the normalization condition $u_{\mu} u^{\mu}=-1$, which holds at any perturbative order, so that

$$
\Delta^{n}\left[u_{\mu} g^{\mu \nu} u_{\nu}\right]=0 .
$$

By applying the Leibniz rule $n$ times and separating the terms linear in perturbations of order $n$, we can rewrite this equation as

$$
\begin{aligned}
- & 2^{\{n\}} u_{\mu} u^{\mu}-u_{\mu} u_{\nu} \Delta^{n}\left[g^{\mu \nu}\right] \\
= & \sum_{k=1}^{n-1} \sum_{i=k}^{n} \frac{n !}{k !(i-k) !(n-i) !} \Delta^{k}\left[g^{\mu \nu}\right]^{\{i-k\}} u_{\mu}{ }^{\{n-i\}} u_{\nu} \\
& +\sum_{i=1}^{n-1}\left(\begin{array}{c}
n \\
i
\end{array}\right)\{i\} u_{\mu}{ }^{\{n-i\}} u^{\mu} \equiv\{n\} Y .
\end{aligned}
$$

On the other hand, the $n$th order perturbation of the inverse metric is given by [23]

$$
\begin{aligned}
\Delta^{n}\left[g^{\mu \nu}\right]= & \sum(-1)^{m} \frac{n !}{k_{1} ! \ldots k_{m} !}{ }^{\left\{k_{1}\right\}} h^{\mu \alpha\left\{k_{2}\right\}} h_{\alpha}{ }^{\beta} \ldots \\
& \times{ }^{\left\{k_{m-1}\right\}} h_{\sigma}{ }^{\rho\left\{k_{m}\right\}} h_{\rho}{ }^{\nu},
\end{aligned}
$$

where this sum extends over all sorted partitions of $n$ such that $k_{1}+\cdots+k_{m}=n$ with $m \leq n$. In spherical symme- try the background four-velocity has no angular components $\left(u^{a}=0\right)$, so that the constraint (37) only contains nonangular components of the $n$th perturbation of the fourvelocity. Since the quantity ${ }^{\{n\}} \Upsilon$ defined in Eq. (37) contains only perturbations up to order $n-1$, we can make the following ansatz:

$$
{ }^{\{n\}} u^{A} \equiv \frac{1}{2}{ }^{\{n\}} \Upsilon u^{A}-\frac{1}{2} \Delta^{n}\left[g^{A B}\right] u_{B}+{ }^{\{n\}} \tilde{\gamma} n^{A},
$$

that reproduces the first-order formula used in Ref. [45] and trivially satisfies the constraint (37). In this way, the three independent components of the perturbations of the four-velocity are encoded in the function ${ }^{\{n\}} \tilde{\gamma}$, which will be expanded in a harmonic series with coefficients denoted by ${ }^{\{n\}} \gamma_{l}^{m}$, and the two angular components ${ }^{\{n\}} u^{a}$, with harmonic coefficients ${ }^{\{n\}} \alpha_{l}^{m}$ and ${ }^{\{n\}} \beta_{l}^{m}$.

The object ${ }^{\{n\}} Y$, polynomial in lower-order perturbations and vanishing for $n=1$, is an example of a highorder perturbation source. These sources, whose computation is one of the main objectives in this article, all have a similar internal structure, which we now illustrate at the second-order perturbative level.

We begin by decomposing the four-velocity at first order (cf. [45]),

$$
\begin{aligned}
{ }^{\{1\}} u_{\mu} d x^{\mu} \equiv & \sum_{l, m}\left({ }^{\{1\}} \gamma_{l}^{m} n_{A}+\frac{1}{2}{ }^{\{1\}} \mathcal{K}_{l A B}^{m} u^{B}\right) Z_{l}^{m} d x^{A} \\
& +\sum_{l, m}\left({ }^{\{1\}} \alpha_{l}^{m} Z_{l a}^{m}+{ }^{\{1\}} \beta_{l}^{m} X_{l a}^{m}\right) d x^{a} .
\end{aligned}
$$

We recall our discussion below Eq. (9) and emphasize that all terms in this equation can be considered as gauge invariant.

At second order, we obtain quadratic first-order terms which arise from the right-hand side of Eq. (39) and the perturbation is given by 


$$
\begin{aligned}
& { }^{\{2\}} u_{\mu} d x^{\mu} \equiv \sum_{l, m}\left({ }^{\{2\}} \gamma_{l}^{m} n_{A}+\frac{1}{2}{ }^{\{2\}} \mathcal{K}_{l A B}^{m} u^{B}+\sum_{\bar{l}, \hat{l}}(\epsilon) \mathcal{U}_{\bar{l} \hat{l} l}^{\bar{m} \hat{m} m}{ }_{A}\right) \\
& \times Z_{l}^{m} d x^{A}+\left({ }^{\{2\}} \alpha_{l}^{m} Z_{l a}^{m}+{ }^{\{2\}} \beta_{l}^{m} X_{l a}^{m}\right) d x^{a} .
\end{aligned}
$$

Here we have defined the alternating sign

$$
\epsilon \equiv(-1)^{\bar{l}+\hat{l}-l},
$$

which is a function of the labels $\bar{l}, \hat{l}, l$, even though we omit these in the notation, and the sum

$$
\sum_{\bar{l}, \hat{l}} \equiv \sum_{\hat{l}=0}^{\infty} \sum_{\bar{l}=0}^{\infty} \sum_{\hat{m}=-\hat{l}}^{\hat{l}} \sum_{\bar{m}=-\bar{l}}^{\bar{l}},
$$

with the restrictions $|\bar{l}-\hat{l}| \leq l \leq|\bar{l}+\hat{l}|$ and $\bar{m}+\hat{m}=m$ for given $l$ and $m$. The source terms ${ }^{(\epsilon)} \mathcal{U}$ can be classified into two groups; those of the form ${ }^{(+)} \mathcal{U}$ are formed by polar $\times$ polar or axial $\times$ axial terms, whereas the terms ${ }^{(-)} \mathcal{U}$ consist of mixed polar $\times$ axial terms. This structure becomes clear in the explicit form of the sources,

$$
\begin{aligned}
& { }^{(+)} \mathcal{U}_{\bar{l} \hat{l} \hat{l} l m}^{\bar{m} m}{ }_{A}=E_{0 \hat{l} \hat{m} l}^{0 \bar{m} \bar{m}}\left\{u_{A}\left[\hat{\gamma} \bar{\gamma}-\hat{\gamma} \overline{\mathcal{K}}_{B C} n^{B} u^{C}+\frac{1}{4} \hat{\mathcal{K}}^{B D} \overline{\mathcal{K}}_{C D} u_{B} u^{C}-\hat{\mathcal{K}}^{B C} \overline{\mathcal{K}}_{C D} u_{B} u^{D}\right]-u_{B} \hat{\mathcal{K}}^{B C} \overline{\mathcal{K}}_{A C}\right\} \\
& -\frac{2}{r^{2}} E_{1 \hat{l} \hat{m} l}^{-1 \bar{m}}\left\{u_{A}\left[\hat{\alpha} \bar{\alpha}+\hat{\beta} \bar{\beta}+\hat{\kappa}_{B} u^{B} \bar{\kappa}_{C} u^{C}-2 \hat{\kappa}_{B} u^{B} \bar{\beta}-\hat{\kappa}_{B} \bar{\kappa}_{C} u^{B} u^{C}\right]-\hat{\kappa}_{B} u^{B} \bar{\kappa}_{A}\right\}, \\
& { }^{(-)} \mathcal{U}_{\bar{l} \hat{l} l}^{\bar{m} \hat{l} m}{ }_{A}=\frac{4 i}{r^{2}} E_{1 \hat{l} \hat{m} l}^{-1 \bar{m}} u_{A}\left\{\hat{\alpha} \bar{\beta}+\bar{\alpha} \hat{\kappa}^{B} u_{B}\right\} \text {. }
\end{aligned}
$$

Here, the caret and bar denote first-order harmonic components with harmonic labels $(\hat{l}, \hat{m})$ and $(\bar{l}, \bar{m})$, respectively. Even though these individual source terms are not symmetric under the interchange $(\hat{l}, \hat{m}) \leftrightarrow(\bar{l}, \bar{m})$, their sum of type (43) becomes symmetric in (41). In order to save space we will follow this notation for the explicit form of source terms quadratic in the first-order perturbations throughout the remainder of this work.

From now on we will simplify our notation for the firstand second-order case by dropping the labels $n=1$ and $n=2$. The perturbative order will become clear from the context in the following equations. Note also that the expressions for the first-order perturbations can always be reconstructed from their second-order counterparts by dropping the quadratic source terms.
With the perturbations of the four-velocity given by Eqs. (40) and (41), we can perform the harmonic decomposition of the second-order perturbation of the energymomentum tensor (32). The axial components are given by

$$
\begin{gathered}
\tilde{\psi}_{l A}^{m}=\beta_{l}^{m}(p+\rho) u_{A}-i \sum_{\bar{l}, \hat{l}}^{(-\epsilon)} \mathcal{E}_{\bar{l} \hat{l} l}^{\bar{m} m}{ }_{A}, \\
\psi_{l}^{m}=-i \sum_{\bar{l}, \hat{l}}{ }^{(-\epsilon)} \mathcal{E}_{\bar{l} \hat{l} l}^{\bar{m} \hat{m} m},
\end{gathered}
$$

where $\mathcal{E}$ are first-order quadratic sources that will be given below. Similarly, we obtain the polar part

$$
\begin{aligned}
\Psi_{l A B}^{m}= & (\rho+p)\left[\gamma_{l}^{m}\left(u_{A} n_{B}+n_{A} u_{B}\right)+\frac{1}{2}\left(\mathcal{K}_{l A C}^{m} u_{B}+\mathcal{K}_{l B C}^{m} u_{A}\right)\right] u^{C}+p \mathcal{K}_{l A B}^{m}+\rho \omega_{l}^{m}\left(u_{A} u_{B}+c_{s}^{2} n_{A} n_{B}\right) \\
& +C \rho \sigma_{l}^{m} n_{A} n_{B}+\sum_{\bar{l}, \hat{l}}^{(\epsilon)} \mathcal{E}_{\bar{l} \hat{l} \hat{m} m}^{\bar{m} m}{ }_{A B},
\end{aligned}
$$$$
\Psi_{l A}^{m}=(p+\rho) \alpha_{l}^{m} u_{A}+\sum_{\bar{l}, \hat{l}}^{(\epsilon)} \mathcal{E}_{\bar{l} \hat{l} l m}^{\bar{m} \hat{m}{ }_{A}},
$$$$
\tilde{\Psi}_{l}^{m}=p \mathcal{K}_{l}^{m}+c_{s}^{2} \rho \omega_{l}^{m}+C \rho \sigma_{l}^{m}+\sum_{\bar{l}, \hat{l}}^{(\epsilon)} \tilde{\mathcal{E}}_{\bar{l} \hat{l} l}^{\bar{m} \hat{m} m},
$$

$$
\Psi_{l}^{m}=\sum_{\bar{l}, \hat{l}}^{(\epsilon)} \mathcal{E}_{\bar{l} \hat{l} l}^{\bar{m} \hat{l} m}
$$

We note that the polarity sign, that appears as a left superindex of the axial sources is $-\epsilon$, whereas that of the polar sources is $\epsilon$. Also, some source terms appear in the same form in the polar and axial equations as, for example, on the right-hand sides of Eqs. (46) and (49) or Eqs. (47) and (51). This is a general feature of quadratic sources in second-order perturbation theory; see for example [23].

We conclude this discussion by giving the sources explicitly in terms of first-order perturbations and $E$-coefficients, 


$$
\begin{aligned}
& { }^{(+)} \mathcal{E}_{\bar{l} \hat{l} l}^{\bar{m} \hat{m} m}{ }_{A B}=\frac{4}{r^{2}}(p+\rho) E_{1 \hat{l} \hat{m} l}^{-1 \bar{m}}\left\{\hat{\kappa}^{C} \bar{\kappa}_{(A} u_{B)} u_{C}-u_{A} u_{B}\left[\hat{\alpha} \bar{\alpha}+\hat{\beta} \bar{\beta}-2 \hat{\beta} \bar{\kappa}_{C} u^{C}\right]\right\} \\
& +E_{0 \hat{l} \hat{m} l}^{0 \bar{l} l}\left\{2 \rho c_{s}^{2} \hat{\omega} \overline{\mathcal{K}}_{A B}+2 \rho \bar{\gamma}\left[C \hat{\sigma}+\hat{\omega}\left(1+c_{s}^{2}\right)\right]\left(n_{B} u_{A}+n_{A} u_{B}\right)+2 C \rho \hat{\sigma}\left(\overline{\mathcal{K}}_{A}{ }^{C} n_{B} n_{C}+\overline{\mathcal{K}}_{C[B} u_{A]} u^{C}\right)\right. \\
& +2 \rho \hat{\omega}\left(1+c_{s}^{2}\right) \overline{\mathcal{K}}_{C(B} u_{A)} u^{C}+(p+\rho)\left[2 \bar{\gamma} \hat{\gamma}\left(n_{A} n_{B}+u_{A} u_{B}\right)-2 \hat{\mathcal{K}}^{C D} \overline{\mathcal{K}}_{D(A} u_{B)} u_{C}+2 \bar{\gamma} \hat{\mathcal{K}}_{C(A} n_{B)} u^{C}\right. \\
& \left.-2 \bar{\gamma} \hat{\mathcal{K}}_{C D} n^{C} u^{D} u_{A} u_{B}+\frac{1}{2} \hat{\mathcal{K}}_{A C} u^{C} \overline{\mathcal{K}}_{B D} u^{D}-\frac{3}{2} \hat{\mathcal{K}}_{C}{ }^{F} \overline{\mathcal{K}}_{D F} u^{C} u^{D} u_{A} u_{B}\right] \\
& \left.+\rho n_{A} n_{B}\left(\bar{\sigma} \hat{\sigma} \frac{\partial C}{\partial s}+2 \bar{\omega} \hat{\sigma} \frac{\partial c_{s}^{2}}{\partial s}+\rho \bar{\omega} \hat{\omega} \frac{\partial c_{s}^{2}}{\partial \rho}\right)\right\} \\
& { }^{(-)} \mathcal{E}_{\bar{l} \hat{l} l \hat{m} m}^{\bar{m} m}{ }_{A B}=\frac{8 i}{r^{2}} E_{1 \hat{l} \hat{m} l}^{-1 \bar{m}} \bar{\alpha}\left(\hat{\kappa}^{C} u_{C}-\hat{\beta}\right)(p+\rho) u_{A} u_{B} \\
& { }^{(+)} \mathcal{E}_{\bar{l} \hat{l} \hat{l} l}^{\bar{m} m}{ }_{A}=2 E_{0 \hat{l} \hat{m} l}^{1 \bar{l} \bar{\alpha}} \bar{\alpha}\left\{(p+\rho)\left[\hat{\gamma} n_{A}+\frac{1}{2} \hat{\mathcal{K}}_{A B} u^{B}\right]+\rho \hat{\omega}\left(1+c_{S}^{2}\right) u_{A}+C \rho \hat{\sigma} u_{A}\right\} \\
& { }^{(-)} \mathcal{E}_{\bar{l} \hat{l} l}^{\bar{m} \hat{m} m}{ }_{A}=2 i E_{0 \hat{l} \hat{m} l}^{1 \bar{l} \bar{m}}\left\{(p+\rho)\left[\hat{\gamma} n_{A}+\frac{1}{2} \hat{\mathcal{K}}_{A B} u^{B}\right] \bar{\beta}+\rho \bar{\beta}\left[\left(c_{s}^{2}+1\right) \hat{\omega}+C \hat{\sigma}\right] u_{A}+\rho\left(C \hat{\sigma}+c_{s}^{2} \hat{\omega}\right) \bar{\kappa}_{A}\right\}, \\
& { }^{(+)} \tilde{\mathcal{E}}_{\bar{l} \hat{l} l}^{\bar{m} \hat{l} m}=-\frac{2}{r^{2}}(p+\rho) E_{1 \hat{l} \hat{m} l}^{-1 \bar{l} m}\{\hat{\alpha} \bar{\alpha}+\hat{\beta} \bar{\beta}\}+2 \rho E_{0 \hat{l} \hat{m} l}^{0 \bar{m} l}\left\{\hat{\mathcal{K}}\left(\bar{\omega} c_{s}^{2}+\bar{\sigma} C\right)+\hat{\omega} \bar{\sigma} \frac{\partial c_{s}^{2}}{\partial s}+\frac{1}{2} \hat{\sigma} \bar{\sigma} \frac{\partial C}{\partial s}+\frac{\rho}{2} \hat{\omega} \bar{\omega} \frac{\partial c_{s}^{2}}{\partial \rho}\right\}, \\
& { }^{(-)} \tilde{\mathcal{E}}_{\bar{l} \hat{l} l}^{\bar{m} \hat{m} m}=\frac{4 i}{r^{2}} E_{1 \hat{l} \hat{m} l}^{-1 \bar{l} m}(p+\rho) \hat{\alpha} \bar{\beta} \\
& { }^{(+)} \mathcal{E}_{\bar{l} \hat{l} l}^{\bar{m} \hat{m} m}=2 E_{1 \hat{l} \hat{m} l}^{1 \bar{l} \bar{m}}(\hat{\alpha} \bar{\alpha}-\hat{\beta} \bar{\beta})(p+\rho) \\
& { }^{(-)} \mathcal{E}_{\bar{l} \hat{l} l}^{\bar{m} \hat{m} m}=4 i E_{1 \hat{l} \hat{m} l}^{1 \bar{m} \bar{\alpha}} \hat{\alpha} \bar{\beta}(p+\rho) .
\end{aligned}
$$

\section{SECOND-ORDER EVOLUTION EQUATIONS}

We now turn our attention to the evolution equations. The results derived in the previous sections facilitate the formulation of the evolution equations at arbitrary order. In order to keep the complexity of the equations in this article at a manageable level, however, we will restrict this discussion to the simplest nonlinear case, the evolution equations for second-order perturbations.

Note again that our calculation represents a generalization of the first-order results of Ref. [45]. Because the equations at second order share the linear part with those at first order, our main task will be the computation of the additional source terms quadratic in the first-order perturbations.

In the Einstein equations these quadratic source terms contain two parts: (i) terms arising from the second-order perturbation of the Einstein tensor which were denoted by (є) $S_{\bar{l} \hat{l} l}^{\bar{m} \hat{m} m}$ in their original derivation in Ref. [23]; and (ii) sources ${ }^{(\epsilon)} \mathcal{E}_{\bar{l} \hat{l} l}^{\bar{m} \hat{m} m}$, obtained from the perturbation of the energy-momentum tensor, which have been given explicitly in Eqs. (52)-(59) for the case of perfect fluid matter. It is convenient to combine those two types as

$$
{ }^{(\epsilon)} \mathcal{P}_{\bar{l} \hat{l} l}^{\bar{m} \hat{m} m} \equiv 8 \pi^{(\epsilon)} \mathcal{E}_{\bar{l} \hat{l} \hat{l} l}^{\bar{m} \hat{m} m}-{ }^{(\epsilon)} S_{\bar{l} \hat{l} l}^{\bar{m} \hat{m} m},
$$

for all tensorial, vectorial, and scalar sources. The perturbative evolution equations for the matter can be computed straightforwardly by direct perturbation of their nonlinear counterparts. For simple systems, including perfect fluids, they can also be obtained from the equations of energymomentum conservation. In particular, the quadratic sources for the fluid equations of motion can be computed from the $I_{\bar{l} \hat{l} l}^{\bar{m} \hat{m} m}$ sources of energy-momentum conservation defined in Eqs. (100-102) of Ref. [23].

Once again, in order to simplify our notation, we will omit from now on the harmonic labels $\{l, m, \hat{l}, \hat{m}, \bar{l}, \bar{m}\}$ from the source names and merely write ${ }^{(\epsilon)} \mathcal{P},{ }^{(\epsilon)} \mathcal{P}_{A},{ }^{(\epsilon)} \mathcal{P}_{A B}$, and so on. We also omit the labels $\{l, m\}$ from the second-order perturbations. They will be distinguished from those at first order because the latter are always denoted with hat or bar overscripts.

Let us summarize the variables that will be used to describe the different perturbative degrees of freedom. The fundamental variables describing density $\omega$, entropy $\sigma$, and velocity perturbations $\{\alpha, \beta, \gamma\}$, have already been defined in Eqs. (34), (35), and (41), respectively. All other 
tensorial perturbative variables will be decomposed in the natural background frame provided by the fluid velocity $\left(u^{A}, n^{A}\right)$ as follows. We encode the axial metric information in the scalars $\{\delta, \lambda\}$, defined as

$$
\kappa_{A} \equiv \delta u_{A}+\lambda n_{A},
$$

whereas the polar tensor $\mathcal{K}_{A B}$ is decomposed in three scalar functions,

$$
\begin{aligned}
\mathcal{K}_{A B} \equiv & \eta\left(n_{A} n_{B}-u_{A} u_{B}\right)+\phi\left(u_{A} u_{B}+n_{A} n_{B}\right) \\
& +\psi\left(u_{A} n_{B}+n_{A} u_{B}\right) .
\end{aligned}
$$

Note that the scalar $\psi$ introduced here, and that will be used in the rest of the article, is different from the harmonic function $\psi$ in the decomposition of the perturbations of the energy-momentum tensor (13). In addition, for future convenience, the metric component function $\phi$ will be replaced by the new variable $\chi$, defined by

$$
\phi \equiv \chi+\mathcal{K}-\eta \text {. }
$$

Finally, the fourth polar geometric degree of freedom will be described by the scalar gauge-invariant $\mathcal{K}$.

Regarding the linear evolution equations, we sill separate the polar and axial parts for arbitrary (second-order) harmonic label $l$. As shown in Ref. [45], for those cases with $l \geq 2$, the axial sector reduces to two independent equations, one for the axial gravitational wave $(\Pi$, defined below) and the other for a fluid perturbation $(\beta)$ describing rotation. On the other hand, the polar sector, also for $l \geq 2$, contains four fundamental equations for four variables, respectively, describing the polar gravitational wave $(\chi)$, the sound wave $(\mathcal{K})$, the entropy perturbation $(\sigma)$, and nonrotational tangential fluid motion $(\psi)$. All other metric and fluid perturbations can be reconstructed from those using the constraint equations. In particular, the function $\eta$ turns out to be vanishing in the case $l \geq 2$. The particular cases $l=0,1$ are nonradiative and the corresponding equations present a different structure. In particular it is not possible to construct gauge invariants for $l=0,1$, and we will need to resort to adequate gauge fixing $[31,34]$.

\section{A. Axial perturbations}

\section{The case $l \geq 2$}

There is a single axial fluid perturbation $\beta$ [defined in Eq. (41)], which obeys the following transport equation, equivalent to the axial part of the perturbed equation of energy-momentum conservation (cf. Eq. (99) of Ref. [23]):

$$
\dot{\beta}-c_{s}^{2}(\mu+2 U) \beta=i \sum_{\bar{l}, \hat{l}}^{(-\epsilon)} \mathcal{B} \equiv \frac{i}{p+\rho} \sum_{\hat{l}, \hat{l}}\left\{(-\epsilon) I-\frac{(l-1)(l+2)}{2 r^{2}}(-\epsilon) \mathcal{E}+\frac{1}{r^{2}}\left[r^{2(-\epsilon)} \mathcal{E}_{A}\right]^{\mid A}\right\} .
$$

The source term ${ }^{(\epsilon)} \mathcal{B}$ is given in expanded form as follows:

$$
\begin{aligned}
{ }^{(-)} \mathcal{B}= & i \frac{2(l-1)(l+2)}{r^{2}} \bar{\alpha} \hat{\beta} E_{1 \hat{l} \hat{m} l}^{1 \bar{l} \bar{m}}-i E_{0 \hat{l} \hat{m} l}^{1 \bar{l} \bar{m}}\left\{\bar{\beta}^{\prime}(2 \hat{\gamma}+\hat{\psi})+\bar{\beta}\left(-\frac{1}{c_{s}^{2}}\left(\frac{C s^{\prime} \rho}{\rho+p}+\nu\right)(2 \hat{\gamma}+\hat{\psi})\right.\right. \\
& +\frac{2 \rho}{\rho+p}\left(\dot{\hat{\omega}}+c_{s}^{2} \dot{\hat{\omega}}+C \dot{\hat{\sigma}}\right)+2 \hat{\gamma}^{\prime}+\hat{\psi}^{\prime}+\dot{\hat{\gamma}}+3 \hat{\mathcal{K}}+2 \frac{1+c_{s}^{2}}{\rho+p}(\mu+2 U)\left(\rho C \hat{\sigma}+\rho c_{s}^{2} \hat{\omega}-p \hat{\omega}\right) \\
& \left.\left.+2 W(2 \hat{\gamma}+\hat{\psi})-2(\mu+2 U)\left(\hat{\sigma} \frac{\partial c_{s}^{2}}{\partial s}+\hat{\omega} \rho \frac{\partial c_{s}^{2}}{\partial \rho}\right)\right)\right\}, \\
{ }^{(+)} \mathcal{B}= & \left.\frac{(l-1)(l+2)}{r^{2}} E_{1 \hat{l} \hat{m} \hat{m} l}^{1 \bar{\alpha} \bar{\alpha}} \bar{\alpha}-\hat{\beta} \bar{\beta}\right)+E_{0 \hat{l} m l}^{1 \bar{l} \bar{m} l}\left[\frac{\bar{l}(\bar{l}+1)}{r^{2}} 2 \bar{\beta} \hat{\delta}+2 \bar{\gamma} \hat{\psi}-\frac{2 \rho}{\rho+p} \overline{\mathcal{K}}\left(c_{s}^{2} \hat{\omega}+C \hat{\sigma}\right)\right. \\
& +\frac{\rho}{\rho+p}(2 \hat{\eta}-\hat{\chi}) \bar{\omega}\left(1+c_{s}^{2}\right)-\bar{\alpha}^{\prime}(2 \hat{\gamma}+\hat{\psi})-\bar{\alpha}\left(2 \hat{\gamma}^{\prime}+\hat{\psi}^{\prime}\right)+\dot{\hat{\alpha}}\left((\bar{\chi}-2 \bar{\eta}+\overline{\mathcal{K}})+\frac{2 \rho}{\rho+p}\left(\bar{\omega}+c_{s}^{2} \bar{\omega}+C \bar{\sigma}\right)\right) \\
& +\frac{1}{c_{s}^{2}} \bar{\alpha}\left(\frac{C s^{\prime} \rho}{\rho+p}+\nu\right)(2 \hat{\gamma}+\hat{\psi})+c_{s}^{2} \bar{\alpha}(\mu+2 U)(\hat{\mathcal{K}}+\hat{\chi}-2 \hat{\eta})-2 \bar{\alpha} \dot{\hat{\omega}} \frac{\rho}{\rho+p}\left(1+c_{s}^{2}\right)-2 \bar{\alpha} C \dot{\hat{\sigma}} \frac{\rho}{\rho+p} \\
& +2 \bar{\alpha}(\mu+2 U) \hat{\omega} \frac{p}{\rho+p}\left(1+c_{s}^{2}\right)+\overline{\mathcal{K}} \hat{\omega} \frac{\rho\left(1+3 c_{s}^{2}\right)}{\rho+p}-\bar{\alpha}(\dot{\hat{\chi}}+3 \dot{\hat{\mathcal{K}}})-2 \bar{\alpha}(2 \hat{\gamma}+\hat{\psi}) W \\
& \left.+\frac{\rho C \hat{\sigma}}{\rho+p}(\bar{\chi}-2 \bar{\eta}+3 \overline{\mathcal{K}})-2 \bar{\alpha}(\mu+2 U) \frac{\rho C \hat{\sigma}}{\rho+p}+2 \bar{\alpha}(\mu+2 U)\left(\hat{\sigma} \frac{\partial c_{s}^{2}}{\partial s}+\hat{\omega} \rho \frac{\partial c_{s}^{2}}{\partial \rho}\right)\right\} .
\end{aligned}
$$

In order to simplify this expression we have assumed that the first-order harmonic numbers $\hat{l}$ and $\bar{l}$ are greater or equal to 2 and, thus, both $\hat{\eta}$ and $\bar{\eta}$ are vanishing, as will be made explicit after Eq. (91). We could expand the time derivatives of the first-order perturbations $\alpha, \omega$ and $\sigma$, using their evolution equations, but it does not lead to any simplification. 
At first order the axial perturbation $\beta$ evolves freely on the background, in the sense that it obeys a transport equation in which the axial gravitational wave does not appear. We see in (65) that the source terms ${ }^{(-)} \mathcal{B}$ extend this fact to second order because they do not contain any axial metric perturbations, due to a geometrical cancellation of the $E$-coefficients of $\bar{\alpha} \hat{\delta}$. Such a cancellation does not occur for the ${ }^{(+)} \mathcal{B}$ source terms in (66), which contains a term

$$
E_{0 \hat{l} \hat{m} l}^{1 \bar{l} \bar{m}} \frac{\bar{l}(\bar{l}+1)}{r^{2}} 2 \bar{\beta} \hat{\delta} .
$$

This allows a coupling between the axial gravitational wave $\hat{\delta}$ and a rotational mode $\bar{\beta}$, generating a second-order rotational mode $\beta$ for odd $\hat{l}+\bar{l}-l$. Note that in such a case we have $E_{0 \hat{l} \hat{m} l}^{1 l \bar{m}}=-E_{1 \hat{l} \hat{m} l}^{0 l \bar{m}}$ (see [23]).

At any given perturbative order $n$ the axial gravitational wave is fully described by the Gerlach-Sengupta master scalar

$$
{ }^{\{n\}} \Pi \equiv \epsilon^{A B}\left(\frac{{ }^{\{n\}} \kappa_{A}}{r^{2}}\right)_{\mid B} .
$$

For $l \geq 2$ one of the perturbed Einstein equations provides a simple way to reconstruct the vector $\kappa_{A}$ from the master scalar $\Pi$ :

$$
\begin{aligned}
(l-1)(l+2) \kappa_{A}= & 16 \pi r^{2} \tilde{\psi}_{A}-\epsilon_{A B}\left(r^{4} \Pi\right)^{\mid B} \\
& +2 i r^{2} \sum_{\bar{l}, \hat{l}}^{(-\epsilon)} S_{A},
\end{aligned}
$$

(cf. Eqs. (80) and (D5) of [23]), and hence there is a one-toone correspondence between $\Pi$ and $\kappa_{A}$, assuming the matter perturbations and sources are known. Expanding this relation in the natural frame, as done in (61), results in the following expressions for the component functions:

$$
\begin{aligned}
\delta= & \frac{r^{2}}{(l-1)(l+2)}\left(r^{2} \Pi^{\prime}+4 r^{2} W \Pi+16 \pi \beta(p+\rho)\right. \\
& \left.+2 i \sum_{\hat{l}, \hat{l}}^{(-\epsilon)} \mathcal{P}_{A} u^{A}\right), \\
\lambda= & -\frac{r^{2}}{(l-1)(l+2)}\left(r^{2} \dot{\Pi}+4 r^{2} U \Pi+2 i \sum_{\hat{l}, \hat{l}}^{(-\epsilon)} \mathcal{P}_{A} n^{A}\right) .
\end{aligned}
$$

One further differentiation of (69) gives an evolution equation for the axial sector of the Einstein equations, which at second order is

$$
\begin{gathered}
-\left[\frac{1}{2 r^{2}}\left(r^{4} \Pi\right)^{\mid A}\right]_{\mid A}+\frac{(l-1)(l+2)}{2} \Pi \\
=8 \pi \epsilon^{A B} \tilde{\psi}_{A \mid B}+i \epsilon^{A B} \sum_{\hat{l}, \hat{l}}^{(-\epsilon)} S_{A \mid B} .
\end{gathered}
$$

By expanding the energy-momentum perturbations in terms of the fundamental fluid variables (46), this equation becomes

$$
\begin{aligned}
- & {\left[\frac{1}{2 r^{2}}\left(r^{4} \Pi\right)^{\mid A}\right]_{\mid A}+\frac{(l-1)(l+2)}{2} \Pi } \\
= & 8 \pi(p+\rho) \beta^{\prime}-\frac{8 \pi}{c_{s}^{2}}\left[\rho C s^{\prime}+\nu(p+\rho)\right] \beta \\
& -i \epsilon^{A B} \sum_{\bar{l}, \hat{l}}^{(-\epsilon)} \mathcal{P}_{A \mid B} .
\end{aligned}
$$

The expanded form of this source is too large to be given here.

In summary, the evolution of the second-order axial perturbations for $l \geq 2$ is given by two coupled equations: the matter equation (64) determines the evolution of $\beta$ and the master equation (73) that of the Gerlach and Sengupta scalar $\Pi$. Reconstruction of the metric is facilitated by using Eqs. (70) and (71).

\section{The case $l=1$}

For $l=1$ the right-hand side of the matter equation (64) slightly simplifies but the overall structure remains that of a transport equation for $\beta$,

$$
\begin{aligned}
& (p+\rho)\left[\dot{\beta}-c_{s}^{2}(\mu+2 U) \beta\right] \\
& =i \sum_{\bar{l}, \hat{l}}\left\{(-\epsilon) I+\frac{1}{r^{2}}\left(r^{2(-\epsilon)} \mathcal{E}_{A}\right)^{\mid A}\right\},
\end{aligned}
$$

whose sources are given by (65) and (66) particularizing to $l=1$. However, the treatment of the gravitational part is rather different. The metric perturbation equation (69) is still valid for $l=1$ and now becomes an equation for $\Pi$,

$$
\frac{1}{2 r^{2}} \epsilon_{A B}\left(r^{4} \Pi\right)^{\mid B}=8 \pi \tilde{\psi}_{A}+i \sum_{\bar{l}, \hat{l}}^{(-\epsilon)} S_{A} .
$$

This allows direct integration of $\Pi$ from the knowledge of $\beta$ and the sources, reflecting the nonexistence of dipole gravitational waves. This is clearer by projecting the equation onto the frame vectors $u^{A}$ and $n^{A}$ and expanding the fluid term according to Eq. (46):

$$
\frac{r^{2}}{2}\left(\Pi^{\prime}+4 W \Pi\right)=-8 \pi(p+\rho) \beta-i \sum_{\bar{l}, \hat{l}}^{(-\epsilon)} \mathcal{P}_{A} u^{A},
$$

$$
\frac{r^{2}}{2}(\dot{\Pi}+4 U \Pi)=-i \sum_{\bar{l}, \hat{l}}^{(-\epsilon)} \mathcal{P}_{A} n^{A}
$$

In practice we plan to first integrate $\beta$ using the evolution equation (74) and then obtain $\Pi$ from the constraint (76). The former implies dividing by the factor $(p+\rho)$ which, at least for some equations of state, might vanish at the surface of a fluid ball but the solution can be shown to be nonsingular by power series expansions around the surface. 
A further difference is that now we cannot reconstruct $\kappa_{A}$ from $\Pi$ using the perturbed Einstein equations. We need to integrate the definition (68) of $\Pi$. We proceed as follows. First, we note that any two-dimensional vector can be expressed in terms of two scalar functions $y\left(x^{A}\right)$ and $z\left(x^{A}\right)$ by writing

$$
\frac{1}{r^{2}} \kappa_{A} \equiv y_{\mid A}+\epsilon_{A}^{B} z_{\mid B}=-\left(\dot{y}+z^{\prime}\right) u_{A}+\left(y^{\prime}+\dot{z}\right) n_{A} .
$$

We insert this expression into the definition of the master scalar (68) and obtain

$$
\Pi=-z_{\mid A}^{\mid A}=\ddot{z}+\mu \dot{z}-\nu z^{\prime}-z^{\prime \prime} .
$$

Finally, we note that in the special case $l=1$, the symmetric trace-free tensor $X_{l_{a b}}^{m}$ defined in Eq. (5) vanishes, so that the condition ${ }^{\{n\}} h_{l}^{m}=0$ in Eq. (9) no longer provides a gauge condition. In RW gauge for $l=1$ there thus remains one axial degree of freedom. The function $y$ represents this freedom and a gauge transformation $y \rightarrow$ $y+\xi$ causes a change in the vector $\kappa_{A}$

$$
\kappa_{A} \rightarrow \kappa_{A}+r^{2} \xi_{\mid A}+\cdots .
$$

Here the dots indicate source terms quadratic in first-order perturbations which are given explicitly in Ref. [24]. The resulting change in the component functions $\delta$ and $\lambda$ defined in Eqs. (70) and (71) is

$$
\begin{aligned}
& \delta \rightarrow \delta-r^{2} \dot{\xi}+\cdots, \\
& \lambda \rightarrow \lambda+r^{2} \xi^{\prime}+\cdots .
\end{aligned}
$$

We are therefore able to fix the gauge by eliminating either $\delta$ or $\lambda$ or any combination of the two. It turns out to be most convenient to set $\lambda=0$. Equation (78) then gives $y^{\prime}=-\dot{z}$, which allows us to integrate $y$ from $z$ and the values of $y$ at $r=0$ (a residual gauge corresponding to a generator $\xi$ which obeys $\xi^{\prime}=0$ ). In this gauge, the vector $\kappa_{A}$ is given by

$$
\kappa_{A}=-r^{2}\left(\dot{y}+z^{\prime}\right) u_{A},
$$

where $z$ is obtained from Eq. (79) and $y^{\prime}=-\dot{z}$.

\section{B. Polar perturbations}

Following decomposition (62), we can schematically write the second-order Einstein equations as follows. We indicate in each case the range of values of $l$, the label of the second-order perturbation, for which the various equations hold. For $l \geq 0$,

$$
\begin{aligned}
u^{A} n^{B} E_{A B}[\mathcal{K}]= & -8 \pi(\rho+p) \gamma+4 \pi(\rho-p) \psi \\
& +\sum_{\hat{l}, \hat{l}}^{(\epsilon)} \mathcal{P}_{A B} u^{A} n^{B},
\end{aligned}
$$

$$
u^{A} u^{B} E_{A B}[\mathcal{K}]=8 \pi \rho \omega+8 \pi \rho(\eta-\phi)+\sum_{\hat{l}, \hat{l}}^{(\epsilon)} \mathcal{P}_{A B} u^{A} u^{B},
$$

$$
\begin{gathered}
n^{A} n^{B} E_{A B}[\mathcal{K}]=8 \pi \rho\left(c_{s}^{2} \omega+C \sigma\right)+8 \pi p(\eta+\phi) \\
+\sum_{\bar{l}, \hat{l}}^{(\epsilon)} \mathcal{P}_{A B} n^{A} n^{B} \\
\tilde{E}[\mathcal{K}]=8 \pi \rho\left(c_{s}^{2} \omega+C \sigma\right)+8 \pi p \mathcal{K}+\sum_{\bar{l}, \hat{l}}^{(\epsilon)} \tilde{\mathcal{P}} .
\end{gathered}
$$

For $l \geq 1$,

$$
\begin{gathered}
u^{A} E_{A}[\mathcal{K}]=-(\rho+p) \alpha+\sum_{\hat{l}, \hat{l}}{ }^{(\epsilon)} \mathcal{P}_{A} u^{A}, \\
n^{A} E_{A}[\mathcal{K}]=\sum_{\bar{l}, \hat{l}}{ }^{(\epsilon)} \mathcal{P}_{A} n^{A} .
\end{gathered}
$$

And finally, for $l \geq 2$,

$$
E[\mathcal{K}]=\sum_{\bar{l}, \hat{l}}^{(\epsilon)} \mathcal{P} .
$$

The $E$ are the Gerlach and Sengupta linear differential operators which act on the polar metric perturbations $\left\{\mathcal{K}_{A B}, \mathcal{K}\right\}$ and are defined explicitly in Eqs. (D1-D4) of Ref. [23].

\section{The case $l \geq 2$}

By expanding the part of Eq. (90) which is linear in the second-order perturbations we arrive at the simple expression

$$
\eta=-\sum_{\bar{l}, \hat{l}}^{(\epsilon)} \mathcal{P} .
$$

The scalar $\eta$ is thus given directly in terms of the first-order perturbations. Note that, as commented at the beginning of this section, in the case of first-order perturbation theory there are no source terms and the first-order $\eta$ vanishes. Even though, Eq. (91) does not exist for $l=0,1$ and hence, in these cases, the first-order $\eta$ is nonvanishing.

Motivated by the linearized equations of energymomentum conservation, we can take linear combinations of the system (84)-(89) such that it can be rewritten as

$$
\begin{aligned}
& l \geq 0: \\
& 8 \pi(p+\rho) \gamma=(\dot{\mathcal{K}})^{\prime}+C_{\gamma}+\sum_{\hat{l}, \hat{l}}^{(\epsilon)} C_{\gamma}, \\
& 8 \pi \rho \omega=-\mathcal{K}^{\prime \prime}+2 U \psi^{\prime}+C_{\omega}+\sum_{\hat{l}, \hat{l}}^{(\epsilon)} C_{\omega}, \\
& l \geq 1: \\
& 16 \pi(p+\rho) \alpha=\psi^{\prime}+C_{\alpha}+\sum_{\hat{l}, \hat{l}}^{(\epsilon)} C_{\alpha},
\end{aligned}
$$




$$
\begin{gathered}
-\ddot{\chi}+\chi^{\prime \prime}+2(\mu-U) \psi^{\prime}=S_{\chi}+\sum_{\hat{l}, \hat{l}}^{(\epsilon)} S_{\chi}, \\
-\ddot{\mathcal{K}}+c_{s}^{2} \mathcal{K}^{\prime \prime}-2 c_{s}^{2} U \psi^{\prime}=S_{\mathcal{K}}+\sum_{\bar{l}, \hat{l}}^{(\epsilon)} S_{\mathcal{K}}, \\
-\dot{\psi}=S_{\psi}+\sum_{\hat{l}, \hat{l}}^{(\epsilon)} S_{\psi},
\end{gathered}
$$

where $C_{\gamma}, C_{\omega}, C_{\alpha}, S_{\chi}, S_{\mathcal{K}}$, and $S_{\psi}$ are linear functions of the quantities $\chi, \mathcal{K}$, and $\eta$, their derivatives $\left\{\dot{\chi}, \chi^{\prime}, \dot{\mathcal{K}}, \mathcal{K}^{\prime}, \dot{\eta}, \eta^{\prime}, \eta^{\prime \prime}\right\}$ and of $\psi$ and $\sigma$. These sources are identical to those given in Ref. [45] and are included in Appendix A for completeness. We have also introduced the following linear combinations of quadratic sources:

$$
{ }^{(\epsilon)} C_{\gamma} \equiv{ }^{(\epsilon)} \mathcal{P}_{A B} u^{A} n^{B},
$$

$$
\begin{gathered}
{ }^{(\epsilon)} C_{\omega} \equiv-{ }^{(\epsilon)} \mathcal{P}_{A B} u^{A} u^{B}, \\
{ }^{(\epsilon)} C_{\alpha} \equiv 2^{(\epsilon)} \mathcal{P}_{A} u^{A}, \\
{ }^{(\epsilon)} S_{\chi} \equiv 2^{(\epsilon)} \tilde{\mathcal{P}}+4\left[{ }^{(\epsilon)} \mathcal{P}_{A} n^{A}\right]^{\prime}-2^{(\epsilon)} \mathcal{P}_{A B} n^{A} n^{B} \\
+4(2 \nu-W)^{(\epsilon)} \mathcal{P}_{A} n^{A}, \\
{ }^{(\epsilon)} S_{\mathcal{K}} \equiv\left(-c_{s}^{2} u^{A} u^{B}+n^{A} n^{B}\right)^{(\epsilon)} \mathcal{P}_{A B}+4 W^{(\epsilon)} \mathcal{P}_{A} n^{A}, \\
{ }^{(\epsilon)} S_{\psi} \equiv-2^{(\epsilon)} \mathcal{P}_{A} n^{A} .
\end{gathered}
$$

In order to illustrate the kind of quadratic terms that appear in the above equations, here we also present explicitly those corresponding to the last equation for the variable $\psi$,

$$
\begin{aligned}
& { }^{(+)} S_{\psi}=\frac{2}{r^{2}} E_{2 \hat{l} \hat{m} l}^{-1 \bar{m}}\left\{-\bar{\delta} \dot{\hat{\lambda}}+\hat{\delta} \dot{\bar{\lambda}}+\mu \bar{\delta} \hat{\lambda}-\mu \hat{\delta} \bar{\lambda}+\bar{\delta} \hat{\delta}^{\prime}+\hat{\delta} \bar{\delta}^{\prime}-2 \bar{\lambda} \hat{\lambda}^{\prime}-2 W \hat{\delta} \bar{\delta}+2 W \hat{\lambda} \bar{\lambda}\right\} \\
& +E_{1 \hat{l} \hat{m} l}^{0 \bar{m} l}\left\{2 \overline{\mathcal{K}} \hat{\mathcal{K}}^{\prime}+2 \hat{\mathcal{K}} \overline{\mathcal{K}}^{\prime}+2 \hat{\psi}(\dot{\bar{\chi}}+\dot{\overline{\mathcal{K}}})-2(\dot{\bar{\psi}}+2 \mu \bar{\psi})(\hat{\chi}+\hat{\mathcal{K}})-16 \pi \hat{\alpha}(p+\rho)(2 \bar{\gamma}-\bar{\psi})\right. \\
& +\frac{\bar{l}(1+\bar{l})}{r^{2}}\left[3 \hat{\delta} \dot{\bar{\lambda}}-\bar{\delta} \dot{\hat{\lambda}}+2 \dot{\hat{\delta}} \bar{\lambda}+\mu \bar{\delta} \hat{\lambda}+3 \mu \hat{\delta} \bar{\lambda}+\nu \hat{\lambda} \bar{\lambda}+\bar{\delta} \hat{\delta}^{\prime}+3 \hat{\delta} \bar{\delta}^{\prime}-4 U \hat{\delta} \bar{\lambda}-2(3 W-\nu) \hat{\delta} \bar{\delta}+(2 W+\nu) \hat{\lambda} \bar{\lambda}\right] \\
& \left.+2(\bar{\chi}+\overline{\mathcal{K}})\left(\dot{\hat{\psi}}+\hat{\chi}^{\prime}+\hat{\mathcal{K}}^{\prime}+2 \mu \hat{\psi}-2 W \hat{\chi}-2 W \hat{\mathcal{K}}\right)-2 \bar{\psi}\left(\dot{\hat{\chi}}+\dot{\hat{\mathcal{K}}}+\hat{\psi}^{\prime}-2 W \hat{\psi}\right)\right\}, \\
& { }^{(-)} S_{\psi}=-\frac{2 i}{r^{2}} E_{-1 \hat{l} \hat{m} l}^{2 \bar{m}}\{\bar{\chi} \hat{\lambda}+\overline{\mathcal{K}} \hat{\lambda}+\hat{\chi} \bar{\lambda}+\hat{\mathcal{K}} \bar{\lambda}-\bar{\delta} \hat{\psi}-\hat{\delta} \bar{\psi}\} \\
& -2 i E_{1 \hat{l} \hat{m} l}^{0 \bar{m} \bar{l}}\left\{\hat{\lambda}(\ddot{\bar{\chi}}+3 \ddot{\overline{\mathcal{K}}})-\ddot{\hat{\lambda}}+\dot{\hat{\delta}}^{\prime}(\bar{\chi}+\overline{\mathcal{K}})+\hat{\delta} \dot{\overline{\mathcal{K}}}^{\prime}+\hat{\lambda}\left(2 \dot{\bar{\psi}}^{\prime}+\bar{\chi}^{\prime \prime}\right)+\mu^{\prime} \hat{\lambda} \bar{\psi}-\bar{\psi}\left(\dot{\hat{\lambda}}^{\prime}+\hat{\delta}^{\prime \prime}\right)\right. \\
& +\nu^{\prime}[3 \hat{\lambda}(\bar{\chi}+\overline{\mathcal{K}})-\hat{\delta} \bar{\psi}]-\dot{\nu}[\hat{\delta}(\bar{\chi}+\overline{\mathcal{K}})-2 \hat{\lambda} \bar{\psi}]+\frac{2 M}{r^{3}} \hat{\delta} \bar{\psi}+\frac{\hat{l}(1+\hat{l})}{r^{2}} \overline{\mathcal{K}} \hat{\lambda} \\
& +\frac{\bar{l}(1+\bar{l})}{2 r^{2}}(\overline{\mathcal{K}} \hat{\lambda}-\bar{\chi} \hat{\lambda}+\hat{\chi} \bar{\lambda}+3 \hat{\mathcal{K}} \bar{\lambda}-\bar{\delta} \hat{\psi}+\hat{\delta} \bar{\psi})-8 \pi \rho \hat{\delta} \bar{\psi}+8 \pi(p+\rho)(2 \bar{\gamma}-\bar{\psi}) \hat{\beta} \\
& -4 \pi \hat{\lambda}(5 p+3 \rho)(\bar{\chi}+\overline{\mathcal{K}})+16 \pi \hat{\lambda} \rho\left(c_{s}^{2} \bar{\omega}+C \bar{\sigma}\right)+\overline{\mathcal{K}}^{\prime}[2 \nu \hat{\lambda}-(\mu-2 U) \hat{\delta}]+\hat{\delta}^{\prime}[-\nu \bar{\psi}+\mu-2 U(\bar{\chi}+\overline{\mathcal{K}})] \\
& +(2 U-\mu) \hat{\lambda}^{\prime} \bar{\psi}+2(U+\mu) \hat{\lambda} \bar{\psi}^{\prime}+\hat{\lambda}\left(\mu^{2}+3 \nu^{2}+2 U^{2}\right)(\bar{\chi}+\overline{\mathcal{K}})+(3 \mu+2 U) \dot{\bar{\chi}} \hat{\lambda} \\
& +\dot{\hat{\delta}}\left[\overline{\mathcal{K}}^{\prime}-2(\nu-W)(\bar{\chi}+\overline{\mathcal{K}})\right]+(3 \nu+2 W) \hat{\lambda} \bar{\chi}^{\prime}+\left(3 \mu \hat{\lambda}-\hat{\delta}^{\prime}+8 U \hat{\lambda}+2 W \hat{\delta}\right) \dot{\overline{\mathcal{K}}}+2 W^{2} \hat{\delta} \bar{\psi}+2 \dot{\bar{\psi}} \hat{\lambda}(2 \nu+W) \\
& -\dot{\hat{\lambda}}(\mu \bar{\chi}+\mu \overline{\mathcal{K}}+2 W \bar{\psi})+2 \nu[2 U \hat{\lambda} \bar{\psi}+3 W \hat{\lambda}(\bar{\chi}+\overline{\mathcal{K}})-W \hat{\delta} \bar{\psi}]-\frac{2}{r^{2}}\left[\overline{\mathcal{K}} \hat{\lambda}-r^{2} U W(\hat{\delta}(\bar{\chi}+\overline{\mathcal{K}})+\hat{\lambda} \bar{\psi})\right] \\
& +2 \mu[U \hat{\delta} \bar{\psi}+U(\bar{\chi}+\overline{\mathcal{K}}) \hat{\lambda}+2(\nu+W) \bar{\psi} \hat{\lambda}]\},
\end{aligned}
$$

where, as before, we have also excluded the $\bar{l}, \hat{l}=0,1$ cases by assuming vanishing $\bar{\eta}$ and $\hat{\eta}$ in order to avoid a larger expression.

On the other hand, we note that the entropy perturbation $\sigma$ is the only matter variable appearing in the linear sources for Eqs. (92)-(97). Its evolution follows from entropy conservation $\dot{s}=0$,

$$
\dot{\sigma}+\left(\gamma+\frac{\psi}{2}\right) s^{\prime}=\sum_{i, \hat{l}}^{(\epsilon)} S_{\sigma}
$$

valid for $l \geq 0$, with the source terms 


$$
\begin{aligned}
&{ }^{(+)} S_{\sigma}=-\frac{2}{r^{2}} E_{1 \hat{l} \hat{l} \hat{l} l}^{-1 \bar{m}}\left\{(2 \hat{\beta} \bar{\lambda}+\hat{\delta} \bar{\lambda}) s^{\prime}-2 \hat{\alpha} \bar{\sigma}\right\} \\
&+E_{0 \hat{l} \hat{m} \hat{m} l}^{0 \bar{m}}\left\{2 \hat{\gamma}(\overline{\mathcal{K}}+\bar{\chi}) s^{\prime}-(2 \bar{\gamma}+\bar{\psi}) \hat{\sigma}^{\prime}\right. \\
&+(2 \bar{\eta}-\overline{\mathcal{K}}-\bar{\chi}) \dot{\hat{\sigma}}\}, \\
&{ }^{(-)} S_{\sigma}=-\frac{4 i}{r^{2}} E_{1 \hat{l} \hat{m} l}^{-1 \bar{m} \bar{m}}\left\{\bar{\alpha} \hat{\lambda} s^{\prime}-(\hat{\beta}+\hat{\delta}) \bar{\sigma}\right\} .
\end{aligned}
$$

The second-order variable $\gamma$ in (106) must be replaced by its expression obtained algebraically from the constraint (92).

Therefore, Eqs. (95)-(97) and (106) form a closed system of four evolution equations for the four unconstrained variables $\chi, \mathcal{K}, \psi$, and $\sigma$, for $l \geq 2$. The additional constraint equations (92)-(94) provide algebraic relations to reconstruct the matter perturbations $\gamma, \omega$, and $\alpha$. The energy-momentum conservation equations provide (redundant) evolution equations for these other fluid variables, namely,

$l \geq 0$ :

$$
\begin{gathered}
-\dot{\omega}-\left(1+\frac{p}{\rho}\right)\left(\gamma+\frac{\psi}{2}\right)^{\prime}=S_{\omega}+\sum_{\bar{l}, \hat{l}}^{(\epsilon)} S_{\omega} \\
\left(1+\frac{p}{\rho}\right)\left(\gamma-\frac{\psi}{2}\right)+c_{s}^{2} \omega^{\prime}=S_{\gamma}+\sum_{\hat{l}, \hat{l}}^{(\epsilon)} S_{\gamma} \\
l \geq 1: \quad \dot{\alpha}=S_{\alpha}+\sum_{\hat{l}, \hat{l}}^{(\epsilon)} S_{\alpha}
\end{gathered}
$$

where, as before, we have gathered the nonprincipal part in linear functions $S_{\omega}, S_{\gamma}$ and $S_{\alpha}$ which are given in Appendix A. We have further defined the quadratic sources

$$
\begin{aligned}
\rho \times{ }^{(\epsilon)} S_{\omega} \equiv & \frac{l(l+1)}{r^{2}}{ }^{(\epsilon)} \mathcal{E}_{A} u^{A}+2 U^{(\epsilon)} \tilde{\mathcal{E}}-\frac{1}{r^{2}} \\
& \times\left[r^{2(\epsilon)} \mathcal{E}_{A B}\right]^{\mid B} u^{A}-{ }^{(\epsilon)} I_{A} u^{A}, \\
\rho \times{ }^{(\epsilon)} S_{\gamma} \equiv & \frac{l(l+1)}{r^{2}}(\epsilon) \mathcal{E}_{A} n^{A}+2 W^{(\epsilon)} \tilde{\mathcal{E}}-\frac{1}{r^{2}} \\
& \times\left[r^{2}(\epsilon) \mathcal{E}_{A B}\right]^{\mid B} n^{A}-{ }^{(\epsilon)} I_{A} n^{A}, \\
(p+\rho) \times{ }^{(\epsilon)} S_{\alpha} \equiv{ }^{(\epsilon)} \tilde{\mathcal{E}}-\frac{(l-1)(l+2)}{2 r^{2}}(\epsilon) \mathcal{E}+\frac{1}{r^{2}} & \\
& \times\left[r^{2(\epsilon)} \mathcal{E}^{A}\right]_{\mid A}+{ }^{(\epsilon)} I .
\end{aligned}
$$

It is possible to use Eqs. (94) and (97) to eliminate derivatives of $\psi$ in Eqs. (109) and (110), arriving at the sound wave equation

$$
\begin{aligned}
& l \geq 1: \\
& \quad-\dot{\omega}-\left(1+\frac{p}{\rho}\right) \gamma^{\prime}=\bar{S}_{\omega}+\sum_{\bar{l}, \hat{l}}^{(\epsilon)} \bar{S}_{\omega},
\end{aligned}
$$

$$
\left(1+\frac{p}{\rho}\right) \dot{\gamma}+c_{s}^{2} \omega^{\prime}=\bar{S}_{\gamma}+\sum_{\bar{l}, \hat{l}}^{(\epsilon)} \bar{S}_{\gamma}
$$

Again, the linear sources $\bar{S}_{\omega}$ and $\bar{S}_{\gamma}$ are given in Appendix A.

\section{Polar perturbations with $l=1$}

In the special case $l=1$ there are still three polar degrees of freedom, but the RW gauge only imposes two gauge conditions $\left(H_{A}=0\right)$. In consequence, the variables $\mathcal{K}_{A B}$ no longer serve as gauge invariants but instead change under gauge transformations generated by the vector $\xi_{\mu} d x^{\mu} \equiv r^{2} \xi Z_{a} d x^{a}$ as

$$
\begin{gathered}
\mathcal{K}_{A B} \rightarrow \mathcal{K}_{A B}-\left(r^{2} \xi_{\mid A}\right)_{\mid B}-\left(r^{2} \xi_{\mid B}\right)_{\mid A}+\cdots, \\
\mathcal{K} \rightarrow \mathcal{K}-2 \xi-2 r^{2} v^{A} \xi_{\mid A}+\cdots
\end{gathered}
$$

where the dots indicate source terms quadratic in the firstorder perturbations.

At this point, we follow Campolattaro and Thorne [34] and fix the remaining gauge degree of freedom by demanding $\mathcal{K}$ to vanish. Even then we are still left with the residual freedom of transformations under the restricted class of functions $\xi$ such that $\xi+r^{2} v^{A} \xi_{\mid A}=0$.

We have already mentioned that Eq. (91) is absent for $l=1$, and hence we proceed differently than for $l \geq 2$. We use Eqs. (106), (111), (115), and (116) to evolve the matter perturbations $\{\sigma, \alpha, \omega, \gamma\}$. These equations contain the metric perturbations $\{\eta, \psi, \chi\}$ but not their derivatives. On the other hand, we obtain the metric perturbations from the Einstein equations (92), (94), (96), and (97). With $\mathcal{K}=0$ from the additional gauge freedom, these five equations contain at most first derivatives of $\{\eta, \psi, \chi\}$. Hence, there are five equations for six unknowns: we can obtain $\left\{\dot{\psi}, \psi^{\prime}, \dot{\chi}, \chi^{\prime}\right\}$ but only the following combination of $\dot{\eta}$ and $\eta^{\prime}$ :

$$
D \eta \equiv \frac{r^{\mid A} \eta_{\mid A}}{r^{\mid B} r_{\mid B}}=\frac{1}{r|v|^{2}}\left(W \eta^{\prime}-U \dot{\eta}\right),
$$

where $|v|^{2} \equiv v^{A} v_{A}$. In consequence, we can only integrate $\eta$ on a spatial surface that is everywhere normal to $r=$ const surfaces, and therefore we also integrate the other scalars $\psi$ and $\chi$ in the same form,

$$
\begin{aligned}
r|v|^{2} D \eta= & \operatorname{rhs}(\mathrm{A} 12)-\frac{1}{2} \sum_{\bar{l}, \hat{l}}{ }^{(\epsilon)} S_{\mathcal{K}}+\left(1-c_{s}^{2}\right)^{(\epsilon)} C_{\omega} \\
& \left.-2 U^{(\epsilon)} C_{\alpha}\right\},
\end{aligned}
$$

$$
r|v|^{2} D \chi=\operatorname{rhs}(\mathrm{A} 13)+\sum_{\bar{l}, \hat{l}}\left\{2 U^{(\epsilon)} C_{\alpha}-{ }^{(\epsilon)} C_{\omega}\right\},
$$




$$
r|v|^{2} D \psi=\operatorname{rhs}(\mathrm{A} 14)-\sum_{\bar{l}, \hat{l}}\left\{{ }^{(\epsilon)} C_{\gamma}+W^{(\epsilon)} C_{\alpha}-U^{(\epsilon)} S_{\psi}\right\},
$$

where the notation rhs $(\mathrm{X})$ stands for the right-hand side of Eq. (X) of Ref. [45].

\section{The case $l=0$}

The $l=0$ perturbations are treated in a manner similar to the case $l=1$. We evolve the matter perturbations and then obtain the metric perturbations from the constraints. This is a direct consequence of the absence of radiative degrees of freedom in spherical symmetry.

For $l=0$ we still have four perturbative variables, namely $\chi, \eta, \psi$, and $\mathcal{K}$, and only two gauge degrees of freedom in the gauge-generator vector. The RW gauge does not impose any condition for $l=0$, and hence we need to fix those two gauge degrees in some other way. We do this by extending the gauge used in the previous subsection

$$
\mathcal{K}=0, \quad \psi=\frac{2 U W}{U^{2}+W^{2}}(\eta-\chi) .
$$

In polar-radial coordinates the second condition implies the vanishing of the variable $\psi$. A further feature of this case is that the velocity perturbation $\alpha$ also vanishes.

The evolution procedure is then as follows. The matter perturbations $\sigma, \omega$, and $\gamma$ are evolved by Eqs. (106), (109), and (110), respectively. The latter two equations contain derivatives of the metric perturbations that can be removed by using the perturbed Einstein equations (84)-(86). The resulting equations, as well as the constraint equations for the two nonvanishing metric perturbations $\eta$ and $\chi$ read as follows:

$$
\begin{aligned}
-\dot{\omega}-\left(1+\frac{p}{\rho}\right) \gamma^{\prime}= & \operatorname{rhs}(\mathrm{A} 15)+\sum_{\hat{l}, \hat{l}}\left\{{ }^{(\epsilon)} S_{\omega}-\frac{(p+\rho)}{2 \rho|v|^{2}}\right. \\
& \left.\times\left(U u^{A} u^{B}-W u^{A} n^{B}\right)^{(\epsilon)} \mathcal{P}_{A B}\right\},
\end{aligned}
$$

$$
\begin{aligned}
\left(1+\frac{p}{\rho}\right) \dot{\gamma}+c_{s}^{2} \omega^{\prime}= & \operatorname{rhs}(\mathrm{A} 16)+\sum_{\bar{l}, \hat{l}}\left\{{ }^{(\epsilon)} S_{\gamma}+\frac{(p+\rho)}{2 \rho|v|^{2}}\right. \\
& \left.\times\left(U u^{A} n^{B}-W n^{A} n^{B}\right)^{(\epsilon)} \mathcal{P}_{A B}\right\},
\end{aligned}
$$

$$
\begin{aligned}
r|v|^{2} D \eta= & \operatorname{rhs}(\mathrm{A} 18)+\frac{1}{2|v|^{2}} \sum_{\bar{l}, \hat{l}}\left\{\left(U^{2}+W^{2}\right)\right. \\
& \left.\times\left(u^{A} u^{B}+n^{A} n^{B}\right)-4 U W u^{A} n^{B}\right\}^{(\epsilon)} \mathcal{P}_{A B},
\end{aligned}
$$

$$
\begin{aligned}
r|v|^{2} D \chi= & \operatorname{rhs}(\mathrm{A} 19)+\frac{1}{|v|^{2}} \sum_{\hat{l}, \hat{l}}\left\{\left(U^{2}+W^{2}\right) u^{A} u^{B}\right. \\
& \left.-2 U W u^{A} n^{B}\right\}^{(\epsilon)} \mathcal{P}_{A B},
\end{aligned}
$$

where we have again used the notation $\operatorname{rhs}(\mathrm{X})$ to refer to the right-hand side of different formulas of Ref. [45].

\section{PERTURBATIVE MATCHING TO AN EXTERIOR VACUUM}

So far, we have studied the interior of a perfect fluid system. We now assume that our system is a fluid star surrounded by vacuum, with both regions, interior and exterior, separated by a surface $\Sigma$ where the pressure vanishes. Hence, the background exterior will be Schwarzschild.

The first part of this section describes high-order perturbative matching across any timelike surface in a general background spacetime. The second part will particularize to the case of a spherical fluid interior matched to a vacuum exterior. This generalizes the first-order results of Ref. [54] for the same scenario, and we closely follow their notation.

\section{A. High-order matching conditions}

We describe the matching surface $\Sigma$ as the zero level set of a smooth scalar field $f\left(x^{\mu}\right)$, with arbitrary smooth continuation off the surface. The unit spacelike vector field normal to the surface is defined as

$$
n_{\mu} \equiv \varphi f_{, \mu}, \quad \text { where } \varphi \equiv\left(f_{, \nu} f^{, \nu}\right)^{-1 / 2} .
$$

From this vector we construct the induced metric $i_{\mu \nu} \equiv$ $g_{\mu \nu}-n_{\mu} n_{\nu}$ and the extrinsic curvature $e_{\mu \nu} \equiv n_{\mu ; \alpha} i^{\alpha}{ }_{\nu}$ of the surface $\Sigma$, both of which must be continuous across $\Sigma$ to ensure a smooth matching between the interior and exterior solutions. Before discussing high-order perturbations of these objects we need to address two important issues arising in the perturbative version of the matching problem: index positioning and gauge dependence.

For a generic tensor field $T_{\mu}$ we have

$$
\Delta\left[T_{\mu}\right] \neq g_{\mu \nu} \Delta\left[T^{\nu}\right],
$$

because in general the perturbation of the metric field does not vanish. Imposing continuity on the perturbations of the covariant or contravariant forms of the tensors $i$ and $e$ can therefore lead to different results, and we must decide in advance which are the proper quantities to be used. The first-order discussion in [54] shows that we must use perturbations of the contravariant fundamental forms. Essentially, this is because a contravariant tensor field $T^{\mu \nu \cdots}$ is intrinsic to the surface $\Sigma$ if and only if $T^{\mu \nu \cdots} f_{, \mu}=$ 0 on any of its indices $\mu$. The equivalent condition for a covariant tensor field $T_{\mu \nu \ldots}$, on the other hand, would be $T_{\mu \nu \ldots . .} g^{\mu \alpha} f_{, \alpha}=0$, which involves the ambient metric and therefore introduces additional information not intrinsic to 
the surface. This argument generalizes to higherperturbative orders, as we will later see, and so we will impose continuity of $\Delta^{n}\left[i^{\mu \nu}\right]$ and $\Delta^{n}\left[e^{\mu \nu}\right]$ for all $n$.

Second, we need to deal with the gauge freedom arising from the arbitrariness in our choice of mapping $\Phi$ between the perturbed and the background spacetimes. Under a general perturbation the scalar $f$ will also change, so that the perturbed surface will be described by the level surfaces of $f+\Delta_{\Phi}[f]+\cdots$ (a field on the background manifold), where we have made explicit the gauge $\Phi$ relating the background and perturbed spacetimes. It is possible, therefore, for a perturbed interior point to be located at a coordinate position corresponding to the background exterior. Such a situation can be handled consistently using one-sided derivatives [59]. There remains, however, the question of the correct continuity conditions, because these conditions may not be equivalent if expressed in different gauges. A convenient treatment of this difficulty is possible because there exists a privileged class of gauges $\Omega$, characterized by the condition $\Delta_{\Omega}^{n}[f]=$ 0 which generalizes the first-order surface gauge of [54]. This does not imply that the shape of the surface will not change; indeed, the surface can be highly distorted in the perturbed manifold. However, this kind of mappings between perturbed and background spacetimes will identify any point of the perturbed surface with another point of the background surface. In surface gauge, the matching conditions at any perturbative order are therefore given by the continuity of the induced metric $\Delta_{\Omega}^{n}\left[i^{\mu \nu}\right]$ and the extrinsic curvature $\Delta_{\Omega}^{n}\left[e^{\mu \nu}\right]$.

We emphasize that surface gauge is only used to define the continuity conditions. We can still work using any other gauge as convenient for the interior or exterior problems. The gauge freedom is then handled by constructing gauge invariants associated with surface gauge, that is, combinations of the perturbations in an arbitrary gauge whose values coincide with the result in surface gauge. See [24] for a discussion on the construction of such gauge invariants. This can be achieved by finding the general form of a gauge transformation from arbitrary to surface gauge. Such transformation will be parametrized by the gauge vectors $\left\{{ }^{\{1\}} \xi, \ldots,{ }^{\{n\}} \xi\right\}$ defined by solving the following equation order by order:

$$
\begin{aligned}
0= & \Delta^{n}[f]+\sum_{m=1}^{n} \frac{n !}{(n-m) !} \sum_{\left(K_{m}\right)} \frac{1}{2 !^{k_{2}} \ldots(m !)^{k_{m}} k_{1} ! \ldots k_{m} !} \\
& \times \mathcal{L}_{\{1\} \xi}^{k_{1}} \ldots \mathcal{L}_{\{m\} \xi}^{k_{m}} \Delta^{n-m}[f] .
\end{aligned}
$$

This equation is just the gauge transformation (10) of the $n$th order perturbation of the scalar field $f$ to surface gauge. In particular, solutions at first and second orders are given by

$$
{ }^{\{1\}} \xi_{\mu}=-\Delta[f] n_{\mu},
$$

$$
{ }^{\{2\}} \xi_{\mu}=-\Delta^{2}[f] n_{\mu}+\varphi^{2} \Delta[f] \Delta[f]_{\mu} .
$$

These solutions fix only one of the 4 degrees of freedom in each generator. There thus remain 3 additional degrees of freedom which represent gauge changes among different surface gauges.

Now we can define the gauge-invariant combinations of perturbations via an operator $\bar{\Delta}$ whose action on a background tensor field $T^{\mu}$ is defined as

$$
\begin{aligned}
\bar{\Delta}^{n}\left[T^{\mu}\right] \equiv & \Delta^{n}\left[T^{\mu}\right]+\sum_{m=1}^{n} \frac{n !}{(n-m) !} \sum_{\left(K_{m}\right)} \frac{1}{k_{1} ! \ldots k_{m} !} \\
& \times \frac{1}{2 !^{k_{2}} \ldots(m !)^{k_{m}}} \mathcal{L}_{\{1\} \xi}^{k_{1}} \ldots \mathcal{L}_{\{m\} \xi}^{k_{m}} \Delta^{n-m}\left[T^{\mu}\right],
\end{aligned}
$$

where the gauge vectors $\left\{{ }^{\{1\}} \xi, \ldots,{ }^{\{n\}} \xi\right\}$ are those obtained by solving Eq. (130). Note that the gauge generators depend implicitly on the metric perturbations, so that this formula is nonlinear and highly nontrivial to implement.

The $\bar{\Delta}$ operator has been constructed explicitly so that it obeys $\bar{\Delta}^{n} f=0$ for all $n$. Specifically, this implies

$$
\bar{\Delta}^{n}\left[n_{\mu}\right]=\frac{\bar{\Delta}^{n}[\varphi]}{\varphi} n_{\mu} .
$$

For example, at first order we obtain

$$
\bar{\Delta}[\varphi]=\frac{\varphi}{2} n^{\alpha}\left[n^{\beta\{1\}} h_{\alpha \beta}-2(\varphi \Delta[f])_{\alpha}\right] .
$$

We do not display explicit second- and higher-order formulas here as they are rather complicated and do not contribute to enlighten the discussion.

Equation (134) implies that perturbations of any contravariant tensor intrinsic to the surface $\Sigma$ will also be intrinsic to the perturbed surface. In other words, for a background tensor $T^{\mu}$ with $T^{\mu} n_{\mu}=0$ we also have

$$
\bar{\Delta}^{n}\left[T^{\mu}\right] n_{\mu}=0 .
$$

Note that covariant tensor fields do not share this property.

In summary, we require the barred perturbations of the contravariant fundamental forms $\bar{\Delta}^{n}\left[i^{\mu \nu}\right]$ and $\bar{\Delta}^{n}\left[e^{\mu \nu}\right]$ to be continuous across the surface $\Sigma$ at any perturbative order $n$. Note that these conditions are formulated in any gauge. In contrast to Ref. [54] we therefore do not need to impose surface gauge.

We conclude this discussion by mentioning the alternative approach to perturbative matching introduced by Mukohyama [60] and further developed in [61]. Their results have been given for first-order perturbations and coincide with those presented here and in [54]. Mukohyama and coworkers do not impose surface gauge and include the gauge freedom within the matching surface $\Sigma$. Compared with our approach, the main difference is their use of an abstract copy of the surface $\Sigma$. The matching is performed separately between the boundaries of the 
interior and exterior spacetimes and that new surface. This procedure introduces an additional geometric structure and gives rise to a new type of gauge invariance and, thus, the concept of double gauge invariants. While this can be a convenient feature as, for example, in the reduction from a $D$-dimensional spacetime to a $(D-1)$-dimensional brane, it would complicate our comparatively simple situation of a spherical background, where the geometry of the matching surface is trivial.

\section{B. Matching to vacuum}

In spherical symmetry, the matching conditions can be decomposed into tensor spherical harmonics, which allows us to extract or inject information through the surface independently for the different harmonic components of the metric and matter fields. We have constructed those decomposed continuity conditions for second-order perturbations, but we will not discuss them here, because they involve very large expressions. Again, they coincide with the expressions given in [54] adding sources quadratic in first-order perturbations. In Appendix B we describe in detail and explicitly how to perform the matching for the particular case of a first-order $(l=1, m=0)$ axial and a second-order $(l=2, m=0)$ polar mode. Here we will also mention different ways of describing the exterior perturbations.

The natural exterior frame is given in terms of the radial vector and its orthogonal defined as

$$
r^{A} \equiv a r^{A} \quad t^{A} \equiv-\epsilon^{A B} r_{B},
$$

where the normalization factor $a$ is given in terms of the Hawking mass as $a^{-1} \equiv \sqrt{1-\frac{2 M}{r}}$. In the interior of the star these vectors are related to the fluid frame $\left(u^{A}, n^{A}\right)$ by a hyperbolic rotation.

The axial matching is simplified by the existence of the Gerlach and Sengupta master scalar, which can be defined both in the interior and the exterior without using fluid information, and so it obeys very simple continuity conditions. The polar problem is harder because none of the internal perturbations matches easily with the natural variable describing vacuum perturbations, the Zerilli scalar

$$
\begin{aligned}
{ }^{\{n\}} \Psi \equiv & \frac{2 a^{-2} r^{2}}{6 M+(l-1)(l+2) r}\left(r^{B\{n\}} \mathcal{K}_{A B}-a r^{\{n\}} \mathcal{K}_{\mid A}\right) r^{A} \\
& +r^{\{n\}} \mathcal{K} .
\end{aligned}
$$

This function obeys the following unconstrained evolution equation [62]:

$$
{ }^{\{n\}} \Psi_{\mid A}{ }^{A}-V^{\{n\}} \Psi={ }^{\{n\}} S,
$$

where $V$ is a potential that depends on the scalars $(r, M)$ and on the harmonic label $l$, whereas ${ }^{\{n\}} S$ is a source that depends on lower-order perturbations and vanishes for linear perturbations $n=1$. At second and higher orders one must deal with unexpected divergencies at asymptotic spatial infinity, which must be regularized by modifying the definition of the Zerilli scalar with adequate lowerorder terms, and hence also modifying the form of the source of Eq. (139). See [62] for details on the regularization procedure.

Using the gauge invariance of our perturbative functions, we could use a particular gauge to simplify some equations. For instance, imposing a gauge given by the conditions $G=0, K=0$, and $r^{A} r^{B} H_{A B}=0$, the Zerilli scalar is essentially the harmonic coefficient $r^{A} H_{A}$,

$$
{ }^{\{n\}} \Psi^{\mathrm{BCL}}=-\frac{2 a^{-1} r l(l+1)}{6 M+(l-1)(l+2) r} r^{A\{n\}} H_{A}+{ }^{\{n\}} R,
$$

with ${ }^{\{n\}} R$ being a nonlinear source depending on lowerorder perturbations. The mentioned gauge is similar to the one introduced in Ref. [63] $(G=0, K=0$, and $\left.t^{A} t^{B} H_{A B}=0\right)$. In fact, making use of the perturbative field equations, the linear part of the Zerilli function turns out have the same form.

\section{CONCLUSIONS}

In this work we have presented a general formalism for second-order perturbations of a time-dependent, selfgravitating, spherically symmetric perfect fluid. To this end we have extended the formalism developed in Refs. $[23,24]$, which in turn can be viewed as a generalization of the Gerlach-Sengupta formalism $[43,44]$ to higher orders. The starting point is a $2+2$ decomposition of the background spherical spacetime; the fourdimensional manifold $\mathcal{M}$ is given by the product of a two-dimensional Lorentzian manifold $\mathcal{M}^{2}$ and the twosphere. This separation enables us to fix the standard angular coordinates $(\theta, \phi)$ on the sphere while formulating the fields on $\mathcal{M}^{2}$ in a manifestly covariant form. After decomposition into tensor spherical harmonics we are left with tensors that depend only on the coordinates on $\mathcal{M}^{2}$. We have further defined gauge-invariant objects (for $l \geq 2$ ) in a manner which identifies them with the quantities obtained in RW gauge.

An important feature of the perfect fluid in spherical symmetry is that it defines a complete basis of vectors on the manifold $\mathcal{M}^{2}$, given by the four-velocity of the fluid and its orthogonal. This basis has been used to project all tensorial, background and perturbative, quantities in such a way that one works exclusively with scalar fields. For the case of second-order perturbations, we have explicitly decomposed the perturbations of the energy-momentum into tensor harmonics. By doing so, we have presented for the first time the complete matter sources that appear in the perturbative evolution equations.

Next, we have combined the geometric sources for the Einstein and matter equations, as given in Ref. [23], with the matter sources derived in this article and, thus, obtained 
the complete set of evolution equations. This has been achieved by following the linear analysis of Ref. [45] for both the polar and axial modes and arbitrary harmonic labels $l$. Except for the polar case with $l<2$, we have thus obtained a combined set of evolution equations and constraints. The metric perturbations with $l<2$ have no radiative freedom and are governed exclusively by constraints. In this case, a solution is obtained by first evolving the matter perturbations and then obtaining the metric perturbations from the constraints.

We have also analyzed the problem of matching the interior perturbative perfect fluid solution with the exterior vacuum spacetime at any order. We have demonstrated why tensors are naturally matched in their contravariant form. In surface gauge, defined as the gauge where the perturbed surface is mapped to that of the background, the perturbations of the contravariant fundamental forms must be continuous across the surface. Even though this result is valid as such in surface gauge only, the relations can be transformed to arbitrary gauge so that practical applications are not necessarily restricted to the use of surface gauge.

In summary, we have presented a general formalism capable of studying coupling between any two first-order modes of a spherical time-dependent star. In future work, we plan to apply this formalism to a variety of stellar models including, but not restricted to, the emission of gravitational waves by slowly rotating, collapsing stars.

\section{ACKNOWLEDGMENTS}

This work was supported by the Spanish MICINN Project No. FIS2008-06078-C03-03, the French ANR Grant LISA Science No. BLAN07-1_201699, and by the Deutsche Forschungsgemeinschaft (DFG) via SFB/TR7. U.S. acknowledges support from the Ramón y Cajal Programme of the Spanish Ministry of Education and Sciences (MEC), by FCT-Portugal through Project No. PTDC/FIS/098025/2008 and by grants from the Sherman Fairchild Foundation to Caltech, by NSF Grants No. PHY-0601459, PHY-0652995, and PHY-1057238, and allocations through loni_numrel05, the TeraGrid Advanced Support Program under Grant No. PHY090003, and the Centro de Supercomputación de Galicia (CESGA) under Project No. ICTS-2009-40.

\section{APPENDIX A: POLAR LINEAR SOURCES}

In this appendix we give the linear sources appearing in the polar sector of the evolution equations (92)-(102) and (102)-(116). These sources have been already given in Ref. [45], but have been recomputed in the course of this investigation, as an intermediate step toward the construction of the second-order sources. There is full agreement with the results of Ref. [45].

$$
\begin{aligned}
S_{\chi}=-2 & {\left[2 \nu^{2}+8 \pi \rho-\frac{6 M}{r^{3}}-2 U(\mu-U)\right](\chi+\mathcal{K})+\frac{(l-1)(l+2)}{r^{2}} \chi+3 \mu \dot{\chi}+4(\mu-U) \dot{\mathcal{K}}-(5 \nu-2 W) \chi^{\prime} } \\
- & 2\left[2 \mu \nu-2(\mu-U) W+\mu^{\prime}-\dot{\nu}\right] \psi+2 \eta^{\prime \prime}-2(\mu-U) \dot{\eta}+(8 \nu-6 W) \eta^{\prime} \\
- & {\left[-4 \nu^{2}+\frac{l(l+1)+8}{r^{2}}+8 \nu W+4\left(2 \mu U+U^{2}-4 W^{2}-8 \pi \rho\right)\right] \eta } \\
S_{\mathcal{K}}= & \left(1+c_{s}^{2}\right) U \dot{\chi}+\left[4 U+c_{s}^{2}(\mu+2 U)\right] \dot{\mathcal{K}}-W\left(1-c_{s}^{2}\right) \chi^{\prime}-\left(\nu+2 W c_{s}^{2}\right) \mathcal{K}^{\prime} \\
& -\left[2\left(\frac{1}{r^{2}}-W^{2}\right)+8 \pi p-c_{s}^{2}\left(\frac{l(l+1)}{r^{2}}+2 U(2 \mu+U)-8 \pi \rho\right)\right](\chi+\mathcal{K})-\frac{(l-1)(l+2)}{2 r^{2}}\left(1+c_{s}^{2}\right) \chi \\
& +2\left[-\mu W\left(1-c_{s}^{2}\right)+(\nu+W) U\left(1+c_{s}^{2}\right)\right] \psi+8 \pi C \rho \sigma-2 U \dot{\eta}+2 W \eta^{\prime} \\
& +\left[\frac{l(l+1)+2}{r^{2}}-6 W^{2}+16 \pi p-2 U(2 \mu+U) c_{s}^{2}\right] \eta, \\
\quad S_{\psi}=2 \nu(\chi+\mathcal{K})+2 \mu \psi+\chi^{\prime}-2 \eta(\nu-W)-2 \eta^{\prime}, & \\
C_{\gamma}= & -W \dot{\chi}+U \chi^{\prime}-(\mu-2 U) \mathcal{K} \mathcal{K}^{\prime}+\frac{1}{2}\left[\frac{l(l+1)+2}{r^{2}}+2 U(2 \mu+U)-2 W(2 \nu+W)+8 \pi(p-\rho)\right] \psi-2 U \eta^{\prime} \\
C_{\omega}= & {\left[\frac{l(l+1)}{r^{2}}+2 U(2 \mu+U)-8 \pi \rho\right](\chi+\mathcal{K})-\frac{(l-1)(l+2)}{2 r^{2}} \chi+2[\nu U+(\mu+U) W] \psi+U \dot{\chi}+(\mu+2 U) \dot{\mathcal{K}}^{\prime} } \\
+ & W \chi^{\prime}-2 W \mathcal{K}^{\prime}-2 \eta U(2 \mu+U),
\end{aligned}
$$




$$
\begin{aligned}
& C_{\alpha}=2 \mu(\chi+\mathcal{K})+2 \nu \psi+\dot{\chi}+2 \dot{\mathcal{K}}-2 \eta(\mu+U), \\
& S_{\omega}=\left(1+\frac{p}{\rho}\right)\left[-\frac{l(l+1)}{r^{2}} \alpha+\frac{\dot{\chi}+3 \dot{\mathcal{K}}}{2}+\left(\nu+2 W-\frac{\nu}{c_{s}^{2}}\right)\left(\gamma+\frac{\psi}{2}\right)\right]+(\mu+2 U)\left(c_{s}^{2}-\frac{p}{\rho}\right) \omega \\
& -C\left[\left(\gamma+\frac{\psi}{2}\right) \frac{s^{\prime}}{c_{s}^{2}}-\sigma(\mu+2 U)\right] \\
& S_{\gamma}=\left(1+\frac{p}{\rho}\right)\left[\frac{\chi^{\prime}+\mathcal{K}^{\prime}-2 \eta^{\prime}}{2}+\left[c_{s}^{2}(\mu+2 U)-\mu\right]\left(\gamma-\frac{\psi}{2}\right)\right]-\nu\left(c_{s}^{2}-\frac{p}{\rho}-\frac{\rho+p}{c_{s}^{2}} \frac{\partial c_{s}^{2}}{\partial \rho}\right) \omega-C \sigma^{\prime} \\
& -\sigma\left[C\left(\nu-\frac{s^{\prime}}{c_{s}^{2}} \frac{\partial c_{s}^{2}}{\partial s}\right)+s^{\prime} \frac{\partial C}{\partial s}-\nu\left(1+\frac{p}{\rho}\right) \frac{1}{c_{s}^{2}} \frac{\partial c_{s}^{2}}{\partial s}\right]-\omega s^{\prime}\left[\frac{\partial c_{s}^{2}}{\partial s}-C\left(1+\frac{\rho}{c_{s}^{2}} \frac{\partial c_{s}^{2}}{\partial \rho}\right)\right], \\
& S_{\alpha}=-\frac{\mathcal{K}+\chi}{2}+\eta-c_{s}^{2}(\mu+2 U) \alpha+\frac{c_{s}^{2} \omega+C \sigma}{1+\frac{p}{\rho}} \\
& \bar{S}_{\omega}=\left(1+\frac{p}{\rho}\right)\left[\left(-\frac{l(l+1)}{r^{2}}+8 \pi(\rho+p)\right) \alpha+\frac{\dot{\mathcal{K}}}{2}+(\mu+U) \eta-\mu(\chi+\mathcal{K})\right]+(\mu+2 U)\left(c_{s}^{2}-\frac{p}{\rho}\right) \omega \\
& +C(\mu+2 U) \sigma-\frac{1}{c_{s}^{2}}\left[s^{\prime} C+\left(1+\frac{p}{\rho}\right)\left(\nu-2 W c_{s}^{2}\right)\right]\left(\gamma+\frac{\psi}{2}\right)+\nu\left(1+\frac{p}{\rho}\right)\left(\gamma-\frac{\psi}{2}\right), \\
& \bar{S}_{\gamma}=\left(1+\frac{p}{\rho}\right)\left[\frac{\mathcal{K}^{\prime}}{2}+\left(c_{s}^{2}(\mu+2 U)-\mu\right)\left(\gamma-\frac{\psi}{2}\right)-\mu \psi-\nu(\chi+\mathcal{K})+(\nu-W) \eta\right]-C \sigma^{\prime} \\
& -\sigma C\left[\nu+\frac{s^{\prime}}{C} \frac{\partial C}{\partial s}-\left(\frac{\nu}{C}\left(1+\frac{p}{\rho}\right)+s^{\prime}\right) \frac{1}{c_{s}^{2}} \frac{\partial c_{s}^{2}}{\partial s}\right]+\omega\left[\nu\left(\frac{p}{\rho}-c_{s}^{2}\right)+s^{\prime}\left(C-\frac{\partial c_{s}^{2}}{\partial s}\right)+\left[\nu(\rho+p)+\rho C s^{\prime}\right] \frac{1}{c_{s}^{2}} \frac{\partial c_{s}^{2}}{\partial \rho}\right] .
\end{aligned}
$$

\section{APPENDIX B: EXAMPLE OF MATCHING: FIRST-ORDER AXIAL $(L=1, M=0)$ AND SECOND-ORDER POLAR $(L=2, M=0)$ MODES}

The general expressions for the matching at the stellar surface are quite large. In order to illustrate the procedure in detail, we therefore analyze in this appendix the matching conditions for the particular case of a first-order $\{l=$ $1, m=0\}$ axial mode that, by self coupling, generates the second-order $\{l=2, m=0\}$ polar mode. This is a particularly interesting situation since the nonradiative first-order mode can be interpreted as a slow rotation of the star that produces gravitational radiation through self coupling. For identification of the perturbative order of the individual terms, we use a left superindex $\{1\}$ on first-order perturbations.

The background junction conditions are straightforwardly deduced from continuity of the two first fundamental forms of the surface. The continuous quantities include the scalars $r, \nu$, and $W$ defined in (21) and (23) respectively. Derivatives of continuous quantities in the direction of the fluid velocity $u^{A}$ must also be continuous. This leads to continuity of $U(23)$ and, since both derivatives of $r$ are continuous, to continuity of the Hawking mass (28).
In our particular case, the negative pressure of the fluid $-p$ will be interpreted as the scalar function $f$, since the stellar surface is characterized by $p=0$. The negative sign has been chosen such that $f$ increases with radius $r$. Furthermore the pressure must be continuous across the surface, whereas the energy density $\rho$ may jump there.

The first-order axial matching is simplified by the existence of the Gerlach and Sengupta master scalar (68), which can be defined both in the interior and the exterior without using fluid information. In fact, a first-order junction condition for our particular example is directly obtained from the continuity of the master scalar ${ }^{\{1\}} \Pi$ across the surface. Regarding the axial vector ${ }^{\{1\}} \kappa_{A}$, we only have continuity of the timelike component ${ }^{\{1\}} \kappa_{A} u^{A}$, that has been defined as ${ }^{\{1\}} \delta$ in Eq. (61). In the gauge proposed at the end of subsection IVA $2,{ }^{\{1\}} \lambda \equiv{ }^{\{1}{ }^{1} \kappa_{A} n^{A}=0$, however, the entire axial vector ${ }^{\{1\}} \kappa_{A}$ turns out to be continuous. For simplicity and consistency with that subsection, we will employ this gauge here. A further quantity that must be continuous is given by the combination

$$
{ }^{\{1\}} \Pi^{\prime}+\frac{16 \pi}{r^{2}} \rho^{\{1\}} \beta,
$$

which depends on the axial fluid perturbation ${ }^{\{1\}} \beta$. 
The second-order polar problem is more complicated because none of the internal perturbations matches straightforwardly with the natural variable describing vacuum perturbations, the Zerilli scalar. Decomposing into harmonics the objects $\bar{\Delta}^{2}\left[i^{\mu \nu}\right]$ and $\bar{\Delta}^{2}\left[e^{\mu \nu}\right]$, we find that the following second-order polar quantities must be continuous:

$$
\begin{gathered}
A_{1}=N+S, \\
A_{2}=\chi+2(\nu+W) N-2 \eta, \\
A_{3}=\mathcal{K}-2 N W, \\
A_{4}=\psi+2 \dot{N}-2 U N, \\
A_{5}=\mathcal{K}^{\prime}-2 \eta W+2 U \dot{N} \\
+2\left(4 \pi \rho-2 U^{2}+W^{2}+\nu W-\frac{M+4 r}{r^{3}}\right) N, \\
A_{6}=\chi^{\prime}+2 \mu \psi-2 \eta^{\prime}+2(W+\nu) \eta-2 \ddot{N}+2 U \dot{N} \\
-2\left(4 \pi p+2 \nu^{2}+U^{2}-\nu W+\frac{M-5 r}{r^{3}}\right) N,
\end{gathered}
$$

where the quadratic source

$$
S \equiv-\frac{1}{\sqrt{5 \pi}}^{\{1\}} \delta^{\{1\}} \delta^{\prime},
$$

appears only in the first continuous combination $A_{1}$, and $\eta$ is given algebraically in terms of first-order perturbations (91),

$$
\begin{aligned}
\eta= & \frac{1}{\sqrt{5 \pi}}\left\{8 \pi(p+\rho)^{\{1\}} \beta^{2}+\frac{1}{2}\left[(\nu-2 W)^{\{1\}} \delta+\{1\} \delta^{\prime}\right]^{2}\right. \\
& \left.+\frac{{ }^{11\}} \delta^{2}}{r^{2}}\right\} .
\end{aligned}
$$

Note that some terms of the right-hand side of this expression are continuous, e.g. ${ }^{\{1\}} \delta^{2} / r^{2}$. Hence, when introducing this expression in the definition of the continuous objects (B2)-(B7), those terms can be removed. We note, however, that special care is required for the term $\eta^{\prime}$ in Eq. (B7), because prime derivatives of continuous objects do not have to be continuous. With the exception of $N$, all perturbative objects that form part of the expressions for the continuous objects have been defined in the main body of the article. The remaining variable $N$ is proportional to the gauge invariant associated to the pressure perturbations $p$ by

$$
-\frac{N}{\varphi} \equiv \Delta^{2}[p]+2 \mathcal{L}_{\{1\}} \Delta[p]+\left(\mathcal{L}_{\{2\}}+\mathcal{L}_{\{2\}}^{2}\right) p .
$$

The pressure $p$ must not be confused with the vector ${ }^{\{n\}} p^{\mu}$, whose harmonic components are given in Ref. [24]. Last relation can be written in terms of the second-order gauge- invariant perturbations of the energy-density and entropy,

$$
N \equiv-\varphi \Delta_{\mathrm{GI}}^{2}[p]=-\varphi \rho\left(c_{s}^{2} \omega+C \sigma\right) .
$$

The subindex GI denotes the perturbation expressed in terms of the gauge-invariant objects that, again, has a form equivalent to the perturbation in the RW gauge ${ }^{\{n\}} p^{\mu}=0$. This last equation has no quadratic terms in first-order energy-density and entropy perturbations because both are polar and we assume the first-order perturbations to be exclusively axial. The norm of the normal vector is defined by (128),

$$
\varphi=\left(p_{, A} p^{A}\right)^{-1 / 2}=-\frac{1}{p^{\prime}} .
$$

The second equality holds because the pressure vanishes at the surface at all times; therefore $\dot{p}=0$. The minus sign comes from the fact that $p^{\prime}<0$. On the other hand, making use of the background Euler equation for the fluid (30), we obtain

$$
p^{\prime}=-\nu(p+\rho) .
$$

Combining the last three equations, we finally obtain $N$ in terms of the fluid variables on the surface,

$$
N=-\frac{c_{s}^{2} \omega+C \sigma}{\nu}
$$

As expected, the continuity conditions (B2)-(B7) reduce to the first-order expressions given in [54] in the absence of the source $S$ and the component $\eta$.

\section{Extraction}

In the interior of the star, the relation between the natural exterior frame $\left(r^{A}, t^{A}\right)$, defined in (137), and the fluid frame $\left(u^{A}, n^{A}\right)$ is given by a hyperbolic rotation,

$$
\begin{aligned}
& r^{A}=-a r U u^{A}+a r W n^{A}, \\
& t^{A}=-a r W u^{A}+a r U n^{A} .
\end{aligned}
$$

Replacing this form of the radial vector $r^{A}$ in the definition of the Zerilli function (138) and rewriting it in terms of the fluid variables, one obtains

$$
\begin{aligned}
\Psi= & \frac{2 r^{4}}{6 M+(l+2)(l-1) r}\left\{U \dot{\mathcal{K}}_{\text {out }}+2 U W \psi_{\text {out }}-W \mathcal{K}_{\text {out }}^{\prime}\right. \\
& \left.-2 U^{2} \eta_{\text {out }}+\left(U^{2}+W^{2}\right)\left(\chi_{\text {out }}+\mathcal{K}_{\text {out }}\right)\right\}+r \mathcal{K}_{\text {out }},
\end{aligned}
$$

where primes and dots are always expressed in the fluid frame, and the expression is evaluated just outside the surface. Making use of the continuous quantities (B2)(B7), we arrive at the formula that gives the outside Zerilli function in terms of the fluid inner variables, 


$$
\begin{aligned}
\Psi= & \frac{2 r^{4}}{6 M+(l+2)(l-1) r}\left\{U \dot{\mathcal{K}}_{\text {in }}+2 U W \psi_{\text {in }}-W \mathcal{K}_{\text {in }}^{\prime}\right. \\
& -2 U^{2} \eta_{\text {in }}+\left(U^{2}+W^{2}\right)\left(\chi_{\text {in }}+\mathcal{K}_{\text {in }}\right)-8 \pi r^{2} \rho W N_{\text {in }} \\
& \left.-\frac{W}{r^{2}}\left(-8+l+l^{2}\right)\left(S_{\text {out }}-S_{\text {in }}\right)\right\}+r \mathcal{K}_{\text {in }} .
\end{aligned}
$$

At first order, because of the vanishing of the source $S$, the last term in curly brackets would disappear.

\section{Injection}

Inside the star, the polar variables $\chi$ and $\mathcal{K}$ satisfy a wave equation. Therefore, boundary conditions on the surface of the star must be given for these variables.

The tensor $\mathcal{K}_{A B}$ is decomposed in Schwarzschild coordinates as

$$
\mathcal{K}_{A B} \equiv\left(\begin{array}{ll}
H_{r r} & H_{r t} \\
H_{r t} & H_{t t}
\end{array}\right)
$$

Outside the star the polar metric perturbations $\left\{H_{r r}, H_{r t}, H_{t t}, \mathcal{K}\right\}$ can be recovered once the Zerilli func- tion has been determined [62]. Transfer of information from the exterior to the interior of the star is again obtained from a hyperbolic rotation and use of the continuous quantities (B2)-(B7). Specifically, using the continuous quantities (B3) and (B4), we get

$$
\chi_{\text {in }}=\chi_{\text {out }}-2(\nu+W)\left(S_{\text {out }}-S_{\text {in }}\right)-2\left(\eta_{\text {out }}-\eta_{\text {in }}\right),
$$

$$
\mathcal{K}_{\text {in }}=\mathcal{K}_{\text {out }}+2 W\left(S_{\text {out }}-S_{\text {in }}\right) .
$$

Applying a hyperbolic rotation results in

$$
\begin{aligned}
\chi_{\text {out }}= & \frac{1}{(r-2 M)^{2}}\left\{r^{4} U^{2} H_{t t}+2 r^{3}(r-2 M) U W H_{r t}\right. \\
& \left.+r^{2}(r-2 M)^{2} W^{2} H_{r r}\right\}-\mathcal{K}_{\text {out }} .
\end{aligned}
$$

Hence, we have succeeded in writing the interior variables $\{\mathcal{K}, \chi\}$ in terms of the exterior variables $\left\{\mathcal{K}, H_{t t}, H_{t r}, H_{r r}\right\}$, which in turn can be obtained in terms of the Zerilli function.
[1] B. Abbott et al., Rep. Prog. Phys. 72, 076901 (2009).

[2] F. Beauville et al., Classical Quantum Gravity 25, 045002 (2008).

[3] H. Lueck et al., Classical Quantum Gravity 23, S71 (2006).

[4] D. Tatsumi et al., Classical Quantum Gravity 24, S399 (2007).

[5] M. Punturo et al., Classical Quantum Gravity 27, 084007 (2010).

[6] K. Danzmann and A. Rudiger, Classical Quantum Gravity 20, S1 (2003).

[7] J.A. Font, Living Rev. Relativity 11, 7 (2008), http:// www.livingreviews.org/lrr-2008-7.

[8] F. Pretorius, in Physics of Relativistic Objects in Compact Binaries: From Birth to Coalescence, edited by M. Colpi et al. (Springer, New York, 2009).

[9] C. Ott, Classical Quantum Gravity 26, 063001 (2009).

[10] P. Ajith et al., Classical Quantum Gravity 24, S689 (2007).

[11] P. Ajith et al., Phys. Rev. D 77, 104017 (2008).

[12] T. Damour, A. Nagar, M. Hannam, S. Husa, and B. Brügmann, Phys. Rev. D 78, 044039 (2008).

[13] A. Buonanno et al., Phys. Rev. D 79, 124028 (2009).

[14] Y. Pan et al., Phys. Rev. D 81, 084041 (2010).

[15] E. Poisson, Living Rev. Relativity 7, 6 (2004), http:// www.livingreviews.org/lrr-2004-6.

[16] T. Hinderer and E. E. Flanagan, Phys. Rev. D 78, 064028 (2008).

[17] J. A. González, U. Sperhake, and B. Brügmann, Phys. Rev. D 79, 124006 (2009).

[18] C.O. Lousto, H. Nakano, Y. Zlochower, and M. Campanelli, Phys. Rev. Lett. 104, 211101 (2010).
[19] C. O. Lousto, H. Nakano, Y. Zlochower, and M. Campanelli, arXiv:1008.4360.

[20] C. O. Lousto and Y. Zlochower, arXiv:1009.0292.

[21] K. Kokkotas and B. G. Schmidt, Living Rev. Relativity 2, 2 (1999), http://www.livingreviews.org/lrr-1999-2.

[22] E. Berti, V. Cardoso, and A. O. Starinets, Classical Quantum Gravity 26, 163001 (2009).

[23] D. Brizuela, J.M. Martín-García, and G. A. Mena Marugán, Phys. Rev. D 74, 044039 (2006).

[24] D. Brizuela, J. M. Martín-García, and G. A. Mena Marugán, Phys. Rev. D 76, 024004 (2007).

[25] C. T. Cunningham, R.H. Price, and V. Moncrief, Astrophys. J. 224, 643 (1978).

[26] C. T. Cunningham, R.H. Price, and V. Moncrief, Astrophys. J. 230, 870 (1979).

[27] C. T. Cunningham, R.H. Price, and V. Moncrief, Astrophys. J. 236, 674 (1980).

[28] M. Gabler, U. Sperhake, and N. Andersson, Phys. Rev. D 80, 064012 (2009).

[29] S. Chandrasekhar, Astrophys. J. 140, 417 (1964).

[30] S. Chandrasekhar, Phys. Rev. Lett. 12, 114 (1964).

[31] K. S. Thorne and A. Campolattaro, Astrophys. J. 149, 591 (1967).

[32] R. Price and K. S. Thorne, Astrophys. J. 155, 163 (1969).

[33] K. S. Thorne, Phys. Rev. Lett. 21, 320 (1968).

[34] A. Campolattaro and K. S. Thorne, Astrophys. J. 159, 847 (1970).

[35] J. R. Ipser and K. S. Thorne, Astrophys. J. 181, 181 (1973).

[36] S. Chandrasekhar and V. Ferrari, Proc. R. Soc. A 432, 247 (1991).

[37] J. R. Ipser and R. H. Price, Phys. Rev. D 43, 1768 (1991). 
[38] K. D. Kokkotas and B. F. Schutz, Mon. Not. R. Astron. Soc. 255, 119 (1992).

[39] N. Andersson and K. D. Kokkotas, Phys. Rev. Lett. 77, 4134 (1996).

[40] E. Seidel and T. Moore, Phys. Rev. D 35, 2287 (1987).

[41] E. Seidel, E. S. Myra, and T. Moore, Phys. Rev. D 38, 2349 (1988).

[42] E. Seidel, Phys. Rev. D 42, 1884 (1990).

[43] U. H. Gerlach and U. K. Sengupta, Phys. Rev. D 19, 2268 (1979).

[44] U.H. Gerlach and U. K. Sengupta, Phys. Rev. D 22, 1300 (1980).

[45] C. Gundlach and J. M. Martín-García, Phys. Rev. D 61, 084024 (2000).

[46] T. Harada, H. Iguchi, and M. Shibata, Phys. Rev. D 68, 024002 (2003).

[47] Y. Kojima, Phys. Rev. D 46, 4289 (1992).

[48] S. Chandrasekhar and V. Ferrari, Proc. R. Soc. A 433, 423 (1991).

[49] A. Stavridis, A. Passamonti, and K. Kokkotas, Phys. Rev. D 75, 064019 (2007).

[50] A. Passamonti, A. Stavridis, and K. D. Kokkotas, Phys. Rev. D 77, 024029 (2008).
[51] E. Gaertig and K. D. Kokkotas, Phys. Rev. D 78, 064063 (2008).

[52] C. Krüger, E. Gaertig, and K. D. Kokkotas, Phys. Rev. D 81, 084019 (2010).

[53] E. Gaertig and K. D. Kokkotas, arXiv:1005.5228.

[54] J. M. Martín-García and C. Gundlach, Phys. Rev. D 64, 024012 (2001).

[55] José M. Martín-García, xAct: Efficient Tensor Computer Algebra for Mathematica, http://metric.iem.csic.es/ Martin-Garcia/xAct/.

[56] D. Brizuela, J. M. Martín-García, and G. A. Mena Marugán, Gen. Relativ. Gravit. 41, 2415 (2009).

[57] T. Regge and J. A. Wheeler, Phys. Rev. 108, 1063 (1957).

[58] S. W. Hawking, J. Math. Phys. (N.Y.) 9, 598 (1968).

[59] M. Mars, Classical Quantum Gravity 22, 3325 (2005).

[60] S. Mukohyama, Classical Quantum Gravity 17, 4777 (2000).

[61] M. Mars, F.C. Mena, and R. Vera, Classical Quantum Gravity 24, 3673 (2007).

[62] D. Brizuela, J. M. Martín-García, and M. Tiglio, Phys. Rev. D 80, 024021 (2009).

[63] L. Battiston, P. Cazzola, and L. Lucaroni, Nuovo Cimento B 3, 295 (1971). 